
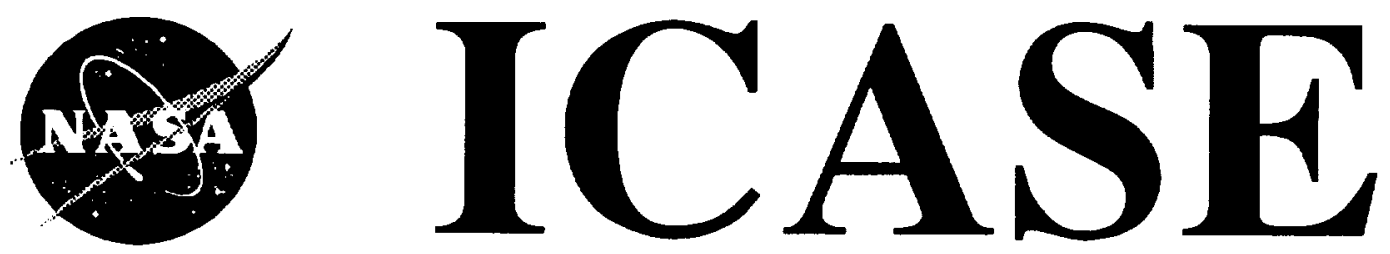

SCALE DISPARITY AND SPECTRAL TRANSFER IN ANISOTROPIC NUMERICAL TURBULENCE

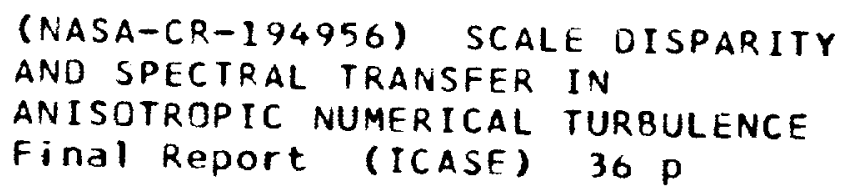

Ye Zhou

P.K. Yeung

James G. Brasseur

Contract NAS1-19480

August 1994

Institute for Computer Applications in Science and Engineering NASA Langley Research Center

Hampton, VA 23681-0001

SRA Operated by Universities Space Research Association 


\title{
Scale disparity and spectral transfer in anisotropic numerical turbulence
}

\author{
Ye Zhou ${ }^{1}$ \\ Institute for Computer Applications in Science and Engineering \\ NASA Langley Research Center, Hampton, VA 23665 \\ P.K. Yeung ${ }^{2}$ \\ School of Aerospace Engineering \\ Georgia Institute of Technology, Atlanta, GA 30332 \\ James G. Brasseur ${ }^{3}$ \\ Department of Mechanical Engineering \\ The Pennsylvania State University \\ University Park, PA 16802
}

\begin{abstract}
To study the effect of cancelations within long-range interactions on local isotropy at the small scales (Waleffe, Phys. Fluids A, 4, 1992), we calculate explicitly the degree of cancelation in distant triadic interactions in the simulations of Yeung \& Brasseur (Phys. Fluids A, 3, 1991) and Yeung, Brasseur \& Wang (to appear, J. Fluid Mech.) using the single scale disparity parameter " $s$ " developed by Zhou (Phys. Fluids A, 5, 1993). In the simulations, initially isotropic simulated turbulence was subjected to coherent anisotropic forcing at the large scales and the smallest scales were found to become anisotropic as a consequence of direct large-small scale couplings. We find that the marginally distant interactions in the simulation do not cancel out under summation and that the development of small-scale anisotropy is indeed a direct consequence of the distant triadic group, as argued by Yeung et al. A reduction of anisotropy at later times occurs as a result of the isotropizing influences of more local energy-cascading triadic interactions. Nevertheless, the distant triadic group persists as an anisotropizing influence at later times. We find that, whereas long-range interactions, in general, contribute little to net energy transfer into or out of a high wavenumber shell $k$, the anisotropic transfer of component energy within the shell increases with increasing scale separation $s$. These results are consistent with results by Zhou, and Brasseur \& Wei (Phys. Fluids, 6, 1994), and suggest that the anisotropizing influences of long range interactions should persist to higher Reynolds numbers. The residual effect of the forced distant triadic group in this low-Reynolds number simulation is found to be forward cascading, on average.
\end{abstract}

\footnotetext{
${ }^{1}$ This research partially supported by the National Aeronautics and Space Administration under NASA Contract No. NAS1-19480 while the author was in residence at the Institute for Computer Applications in Science and Engineering (ICASE), M/S 132C, NASA Langley Research Center, Hampton, VA, 23681-0001

${ }^{2}$ This research was supported by NSF grant CTS 93-07973.

${ }^{3}$ This research was supported by AFOSR/URI grant AFOSR-90-0113, ONR grant N00014-92-J-1417 and ARO/URI grant DAAL03-92-G-0117.
} 



\section{Introduction}

Underlying the high Reynolds number Kolmogorov similarity theory ${ }^{1}$ of 1941 is the implication that interactions among motions at different length scales are statistically dominated by interactions which scale on a single local length scale $r$, so long as $r$ is much smaller than the integral scale $l$. If this is the case, then (a) net energy transfer from motions surrounding scale $r \ll l$ is not directly influenced by integral-scale motions, and (b) the local structure of the motions at scales $r \ll l$ is isotropic. Kolmogorov formulated his theory assuming stationary turbulence in equilibrium at all scales; however, the 1941 hypotheses are commonly applied outside these assumptions.

As pointed out by Batchelor ${ }^{2}$, for example, the assumption of local isotropy has important dynamical implications which extend beyond the statistical implication that the energy cascade is independent of large-scale motions. As defined by Kolmogorov ${ }^{1}$, local isotropy implies that the distribution of velocity differences at scale $r \ll l$ is isotropic, which itself implies that the structure of the motions surrounding $r$ is dynamically decoupled from the structure of the large-scale motions. This dynamical independence between large- and small-scale motions implies that the distribution of small-scale energy and the phase relationships among the small-scale motions are not directly influenced by large-scale structure.

From a Fourier decomposition perspective, the Kolmogorov conjectures lead to the statements that net encrgy transfer from a spectral shell surrounding inverse scale $k \sim 1 / r$ is not directly influenced by large-scale motions, and that the distributions of energy and phase of the Fourier modes in shell $k$ are independent of large-scale structure, when $k \gg 1 / l$. Recently, nonlinear scale interactions and energy transfer in Fourier-space have been studied using direct numerical simulations (DNS) of homogeneous turbulence by several groups ${ }^{3-10}$. Full knowledge of the velocity field in DNS allows the calculation of contributions to the total energy transfer between different scales from pre-defined classes of the nonlinear triadic interactions in Fourier space ${ }^{3}$. In the inertial range, $\mathrm{Zhou}^{6,7}$ found that for isotropic stationary turbulence, consistent with the classical Kolmogorov similarity hypotheses, net energy flux from an inertial shell at radius $k$ is local (occurring between similar scale sizes), but is dominated by "local-to-nonlocal" interactions among scales separated by a decade or less (with the strongest contribution from interactions separated in scale by approximately 1.8 to 5$)$. Scale interactions in the far-dissipation range have also been studied using direct simulations by Zhou ${ }^{7}$, Domaradzki ${ }^{9}$, and Kida et al. ${ }^{10}$.

Consistent with the analysis of $\mathrm{Zhou}^{7}$ in isotropic turbulence, Brasseur \& Wei ${ }^{11}$ found that net energy transfer to/from spectral shells within isolated "chains" of triadic interactions is primarily within local-to-nonlocal triadic interactions with separations in scale of a decade or less. However, they also found that the distant triadic group tends to redistribute energy among Fourier modes within spectral shells in a manner directly related to the structure of the more energetic large-scale motions. Consequently, coherent anisotropic forcing in the energy-containing scales would produce, over time, an anisotropic energy redistribution among small-scale motions as a consequence of direct long-range interactions between the anisotropic energy-containing scales and the smallest scale motions. They argued, therefore, that long-range inertial interactions lead to departures from local isotropy at the smallest dynamical scales, in principle. Waleffe ${ }^{12}$, on the other hand, argued that energy transfer 
within the distant triadic group cancels out, in principle, leaving no net influence of the distant group on the structure of the small scales and local isotropy.

To study the direct influence of the large scales on the distributions of energy and phase within motions surrounding inverse scale scale $k$ far removed from $1 / l$, Yeung and Brasseur $^{4}$ (YB) and Yeung, Brasseur, and Wang $^{5}$ (YBW) performed numerical experiments in which fully-developed isotropic turbulence was subjected to sustained anisotropic forcing in the energy-containing wavenumber range. Marginally distant (highly nonlocal) triadic interactions coupling disparate scales were found to produce, over time, anisotropic redistributions of energy and phase in high wavenumber spectral shells, and consequently an anisotropic small-scale structure. These numerical results, including the later-time evolution of anisotropy under an extended period of forcing, were shown to agree closely with an analysis of the distant triadic energy transfer equations in the asymptotic limit of infinite scale separation ${ }^{5}$. Consequently, although these simulations on $128^{3}$ grids were at moderate Reynolds numbers (20, based on the initial Taylor micro-scale), the DNS analysis and theoretical arguments were taken together to suggest that the distant triadic interactions do not cancel out, and that the statistical independence between large and small scales may not be strictly valid in high Reynolds number turbulence ${ }^{5}$. Nevertheless, $\mathrm{YB}^{4}$ and $\mathrm{YBW}^{5}$ did not directly analyze the extent to which distant triadic interactions cancel out.

This paper explicitly addresses this issue of cancelation within the distant triadic group by re-analyzing the net energy transfer DNS data of $\mathrm{YBW}^{5}$ to show the extent of cancelation using a measure of spectral transfer introduced by Zhou ${ }^{6,7}$ that depends on a single scale disparity parameter, $s$. This new analysis shows explicitly that, whereas the distant triadic group does not contribute to net energy transfer and the forward cascade (as argued by Zhou ${ }^{7}$, and Brasseur \& Wei $^{11}$ ), the marginally distant triadic interactions do not cancel out completely and are in fact the dominant source of the small-scale anisotropy which developed in response to anisotropic forcing at the large scales. We further show that the anisotropizing influence of the distant triadic group, isolated from the nonlocal and local groups, increases with scale disparity, s, suggesting that the influence of long range interactions would persist at higher Reynolds numbers.

In principle, then, the distant triadic group does not entirely cancel out as argued by Waleffe, even though the contribution of this group to the cascade of energy from larger to smaller scales does appear to weaken with increasing scale disparity within the distant group. To understand the dynamics of long-range triadic interactions, it is important to appreciate the distinction between net energy transfer to or from a shell $k$ within the distant triadic group, and the redistribution of energy within the shell $k$ due to distant interactions, which can occur in the absence of net energy transfer into or out of the shell. This distinction is central to understanding this and related studies ${ }^{4-7}$, and for an appreciation of the role of long-range interactions in small scale dynamics in general ${ }^{11}$. It is also important to recognize that the long-range effects which exist in principle may or may not be strong in practice, depending on the state and history of the turbulence ${ }^{5,11}$.

In Sec. II we provide background information relating the studies of $\mathrm{YB}^{4}$ and $\mathrm{YBW}^{5}$ to this new effort, and use of the scale disparity parameter ${ }^{6,7}$ in the analysis of triadic interactions. In Sec. III we present the results relating anisotropy evolution and triadic interactions at two different times in the forced turbulence simulations. Conclusions are summarized in Sec. IV. 


\section{Basic measurements and simulated anisotropic flow fields}

\section{A. Tools of analysis}

Based on a formulation by Kraichnan ${ }^{13}$, Domaradzki \& Rogallo ${ }^{3}$ introduced the energy transfer function

$$
T(k \mid p, q)=\sum_{\mathbf{k}^{\prime} \in \text { shell } k} \sum_{\substack{\mathbf{p}^{\prime} \in \text { shell } p \\ \mathbf{q}^{\prime}=\mathbf{k}^{\prime}-\mathbf{p}^{\prime} \in \text { shell } q}} T\left(\mathbf{k}^{\prime} \mid \mathbf{p}^{\prime}, \mathbf{q}^{\prime}\right),
$$

where

$$
T\left(\mathbf{k}^{\prime} \mid \mathbf{p}^{\prime}, \mathbf{q}^{\prime}\right)=1 / 2 \operatorname{Im}\left[\hat{u}_{i}\left(-\mathbf{k}^{\prime}\right) P_{i l m}\left(\mathbf{k}^{\prime}\right) \hat{u}_{l}\left(\mathbf{p}^{\prime}\right) \hat{u}_{m}\left(\mathbf{q}^{\prime}\right)\right]
$$

is the energy transfer into or out of mode $\mathbf{k}^{\prime}$ due to a single triadic interaction between the mode $\mathbf{k}^{\prime}$ and modes $\mathbf{p}^{\prime}, \mathbf{q}^{\prime} . T(k, p, q)$ is the net energy transfer to/from all Fourier modes in a wavenumber shell centered on radius $k$ due to all triadic interactions (i.e., interactions within wavevector triangles) with one leg of the triad in a shell centered on radius $p$ and the other leg in a shell centered on radius $q$, where the shells are of specified thickness. Here $\hat{u}_{i}\left(\mathbf{k}^{\prime}\right)$ is the Fourier velocity coefficient for wavevector $\mathbf{k}^{\prime}$ in shell $k$, and the projection tensor $P_{i l m}$ is given by $P_{i l m}(\mathbf{k})=k_{m}\left(\delta_{i l}-k_{i} k_{l} / k^{2}\right)+k_{l}\left(\delta_{i m}-k_{i} k_{m} / k^{2}\right)$. Summation is taken in (2) over repeated subscripts, and over all triads $\mathbf{k}^{\prime}=\mathbf{p}^{\prime}+\mathbf{q}^{\prime}$ where the modes $\mathbf{k}^{\prime}$ are within a spherical shell centered on $k$, and modes $\mathbf{p}^{\prime}, \mathbf{q}^{\prime}$ are within shells centered on $p, q$, respectively.

$\mathrm{YB}^{4}$ and $\mathrm{YBW}^{5}$ studied both the net energy transfer $T(k \mid p, q)$ and net component energy transfer $T_{\alpha \alpha}(k \mid p, q)$ (no sum on $\alpha$ ) in their DNS database of anisotropically forced turbulence, where $T_{\alpha \alpha}(k \mid p, q)$ are obtained from Eq. (1) by replacing $i$ with $\alpha$ in (2).

Although $T(k \mid p, q)$ and $T_{\alpha \alpha}(k \mid p, q)$ are fundamental building blocks in the energy transfer process, Zhou ${ }^{6,7}$ has pointed out that identifying the effects of the local, nonlocal or distant triadic groups through $T(k \mid p, q)$ and $T_{\alpha \alpha}(k \mid p, q)$ does not provide the net contributions from these triadic groups. In particular, Zhou observed that the net contributions of all highly nonlocal or distant triadic groups to the net energy transfer $T(k)$ to/from high wavenumber shells $k$ nearly cancel in isotropic turbulence. To explicitly take into account cancelations in net energy transfer to/from shell $k$ resulting from all triadic interactions within a given triadic group based on scale disparity alone, Zhou ${ }^{6,7}$ borrowed from Kraichnan ${ }^{14}$ to introduce the quantity $T(k, s)$, where

$$
s \equiv \frac{\max \left(k^{\prime}, p^{\prime}, q^{\prime}\right)}{\min \left(k^{\prime}, p^{\prime}, q^{\prime}\right)}
$$

is the ratio of the longest to the shortest leg in a triad, giving a direct measure of the disparity of the interacting scales. The contributions of triadic interactions at a given scale disparity parameter $s$ to the net energy transfer a given scale $k$ are characterized by

$$
T(k, s)=\sum_{\mathbf{k}^{\prime} \in \text { shell } k} \sum_{\substack{\mathbf{p}^{\prime}, \mathbf{q}^{\prime} \mid S \\ \mathbf{q}^{\prime}=\mathbf{k}^{\prime}-\mathbf{p}^{\prime}}} T\left(\mathbf{k}^{\prime} \mid \mathbf{p}^{\prime}, \mathbf{q}^{\prime}\right),
$$

which is the partial sum of all triadic interactions involving wavevectors $\mathbf{k}^{\prime}$ in shell $k$ and all other wavevectors $\mathbf{p}^{\prime}$ and $\mathbf{q}^{\prime}=\mathbf{k}^{\prime}-\mathbf{p}^{\prime}$ where the scale disparity $s$ falls into a prescribed 
range. We introduce in this paper the corresponding partial sum with scale parameter $s$ for the net component energy transfer,

$$
T_{\alpha \alpha}(k, s)=\sum_{\mathbf{k}^{\prime} \in \text { shell } k} \sum_{\substack{\mathbf{p}^{\prime}, \mathbf{q}^{\prime} \mid s \\ \mathbf{q}^{\prime}=\mathbf{k}^{\prime}-\mathbf{p}^{\prime}}} T_{\alpha \alpha}\left(\mathbf{k}^{\prime} \mid \mathbf{p}^{\prime}, \mathbf{q}^{\prime}\right) .
$$

Note that all triadic interactions with scale disparity $s$ are included in $T(k, s)$ and $T_{\alpha \alpha}(k, s)$, while $T_{\alpha \alpha}(k \mid p, q)$ and $T(k \mid, p, q)$ include more restrictive groupings of triadic interactions between shell $k$ and shells $p, q$. Both $T_{\alpha \alpha}(k \mid p, q)$ and $T_{\alpha \alpha}(k, s)$ provide useful parameterizations of the scale interactions in energy transfer and are related to the component energy transfer to/from shell $k, T_{\alpha \alpha}(k)$, by

$$
T_{\alpha \alpha}(k)=\sum_{p, q} T_{\alpha \alpha}(k \mid p, q)=\sum_{s} T_{\alpha \alpha}(k, s),
$$

with the corresponding total energy transfers given by summations over $\alpha$. However, because $T_{\alpha \alpha}(k, s)$ and $T(k, s)$ are triadic sums over all interacting scales leaving only the dependence on the scale disparity $s$, these variables provide a more direct measure of scale disparity in net component energy transfer within triadic interactions ${ }^{6,7}$.

In this paper we re-analyze the forced anisotropic turbulence database of $\mathrm{YBW}^{5}$ using the quantities $T_{\alpha \alpha}(k, s)$ and $T(k, s)$.

\section{B. Coherent anisotropic forcing at the large scales}

As described in $\mathrm{YB}^{4}$ and $\mathrm{YBW}^{5}$, anisotropic narrow-band coherent forcing is applied to well resolved $128^{3}$ pseudo-spectral direct simulations of initially isotropic turbulence which is in a state of asymptotic decay. In physical space, the anisotropic forcing is carried out by adding energy and vorticity to an array of 16 large-scale counter-rotating rectilinear vortices, each with vorticity only in the $z$ direction and a two-component two-dimensional velocity field in the $x-y$ plane. In Fourier space nearly all the forcing energy is added to two pairs of Fourier modes with wave-vectors $\mathbf{k}_{F}$ and Fourier velocity coefficients $\hat{\mathbf{u}}\left(\mathbf{k}_{F}\right)$ in the $k_{x}-k_{y}$ plane. The four directly forced modes have wavevector components $( \pm 2, \pm 2,0)$, with magnitude $k_{F}=2 \sqrt{2}$, at the peak in the energy spectrum of the initial isotropic turbulence. In both Fourier and physical spaces, the forced velocity field components $u_{1}$ and $u_{2}$ gain energy directly within the four low wavenumber forced modes, whereas the $u_{3}$ component of velocity, and all Fourier modes other than the forced modes, can be influenced by the forcing only indirectly through nonlinear interscale interactions.

Fig. 1 shows the component energy spectra in the simulations of $\mathrm{YB}^{4}$ and $\mathrm{YBW}^{5}$, at the beginning of forcing and after 1.915 and 3.83 initial eddy-turnover times $\left(T_{E}\right)$. The four directly forced modes appear within the spectral shell centered on $k=3$ in Fig. 1 . After about one eddy-turnover time beyond the initiation of large- scale forcing, the small scales rapidly become anisotropic. However, the structure of this small-scale anisotropy is such that at the high wavenumbers it is $u_{3}$ which has the most energy-in contrast to $u_{1}$ and $u_{2}$ in the forced low wavenumber modes. YBW ${ }^{5}$ showed that the development of small-scale anisotropy begins at the smallest scales first, then progresses up the spectrum from the smallest scales (the highest wavenumbers) to larger scales (lower wavenumbers). 
Concurrently, energy cascarles from the largest (forced) scales to smaller scales. The results of $\mathrm{YBW}^{5}$ indicate that the cascade of energy from the low wavenumber forced shell reduces the level of anisotropy when it reaches higher wavenumber shells. Consequently, the increase in anisotropy at the small scales is followed by a subsequent decrease as energy arrives from the large scales.

The highest wavenumber shell reaches maximum anisotropy in the component spectra at non-dimensional time $t^{*} \equiv t / T_{E}=1.915$ after the beginning of forcing. The component anisotropy subsequently decreases and appears to vanish when $t_{\sim}^{*} 3.5$, even though forcing continues and the large scales are still strongly anisotropic. However, this apparent "return to isotropy" was found to be illusory and a consequence of the inability of second order moments to detect the actual anisotropic structure of the smallest scales. Using third-order moments and three-dimensional visualizations, $\mathrm{YBW}^{5}$ showed that, although the level of anisotropy decreases along with the relative energy level of the forced modes, towards the end of the simulation (at $t^{*}=3.83$ ) the smallest scales retain a significant level of anisotropy. In this paper we analyze results at the two times $t^{*}=1.915$ and $t^{*}=3.83$ using the single scale disparity parameter, $s$.

\section{Analysis and results}

\section{A. Development of anisotropy at the small scales $\left(t^{*}=1.915\right)$}

We focus in this section on the net effects of scale disparity in triadic interactions on the period of development of small-scale anisotropy from large-scale forcing, from the initiation of forcing to $t^{*}=1.915$, the time of maximum component ratio anisotropy at the smallest scales. Consider first Table I, which gives the component contributions to $T(k, s)$ in four representative higher wavenumber shells as a function of scale disparity parameter $s$, where $s$ is divided into octave bands, at the time of maximal anisotropy, $t^{*}=1.915$. The range of $s$ for those triadic interactions with the forced shell are given by $s_{F}$ indicated in Table I for each $k$ shell, determined from

$$
\frac{(k-1 / 2)}{k_{F}} \leq s_{F} \leq \frac{(k+1 / 2)+k_{F}}{k_{F}},
$$

where $k_{F}=2 \sqrt{2}$. Note that the minimum value of $s_{F}$ is when the wavevector $\mathbf{k}$ in shell $k$ (of unit thickness) is the intermediate mode in a triadic interaction, whereas the maximum $s_{F}$ is when $\mathbf{k}$ is the highest wavenumber mode in a triad.

A number of interesting observations are apparent from Table I. For the wavenumber shells such as $k=22$ and $k=44$, where the $s_{F}$ range of forced triads spans two adjacent $s$-bands, the energy transfers $T_{33}(k, s)$ and $T(k, s)$ are dominated by a large positive contribution in the lower $s$-band, followed by a somewhat smaller negative contribution at the next higher band. This behavior is observed in the third and fourth $s$ - bands of $k=22$ and in the fourth and fifth $s$-bands of $k=44$. On the other hand, for wavenumber shells $k=30$ and $k=50$, where the forced triads $\left(s_{F}\right)$ are wholly contained within an $s$-band, a single dominant entry for $T_{33}(k, s)$ and $T(k, s)$ is observed (e.g., the fifth $s$-band of $k=50$ ). The 
explanation for these behaviors will be discussed shortly. Note, at this point, that the dominant interactions in each $k$-shell are nonlocal with the forced shell, and these forced $s$-bands dominate the anisotropic energy transfer within the $k$-shell preferentially increasing energy in the $z$ velocity component relative to the $x-y$ velocity components. Furthermore, as the wavenumber $k$ increases, Table I shows that the dominant interactions become increasingly more nonlocal such that the dominant $s$ - band always contains within it the forced triads, at scale disparity $s_{F}$, coupling that $k$ shell directly with the large-scale forced modes. We conclude that the net effect of nonlocal and distant interactions with the large-scale forced modes is to force that the small scales towards an anisotropic energy distribution in which the spanwise velocity component preferentially gains energy consistent with the high wavenumber spectra of Fig. 1 at $t^{*}=1.915$.

Because $s$ is a direct measure of scale disparity, these observations verify the arguments of $\mathrm{YB}^{4}$ that the more nonlocal and distant interactions are the cause of the development of small-scale anisotropy by large-scale forcing in their simulations ${ }^{4}$. More detailed analysis of the role of the distant triadic group in small-scale evolution is given in Sec. C.

To provide more complete information over a continuous high wavenumber range, Figs. 2 and 3 show the contributions from various $s$-bands to the component transfers $T_{11}(k)$ and $T_{33}(k)$ in the range $40 \leq k<60$ at time $t^{*}=1.915$, and Figs. 4 and 5 show the same contributions in the range $20 \leq k<40$. Because the forcing is symmetric in the $x-y$ plane, $T_{11}(k)$ and $T_{22}(k)$ (not shown) behave similarly.

Consider Figs. 2 and 3 for $40 \leq k<60$. In this $k$ range, the forced triads lie in the range $14 \leq s_{F} \leq 22$, which spans the two $s$-bands $D$ and $E$. In both $T_{11}(k)$ and $T_{33}(k)$, curve $D$ rises to a positive maximum then drops, and curve $E$ drops to a negative minimum then rises back to positive values in the range $42 \leq k<46$. To understand these peaks, consider first those triadic interactions which connect the range $40 \leq k<42$ to the forced modes $k_{F}$. From Eq. 7, the forced triads for $40 \leq k<42$ are in the range $14 \leq s_{F}<16$ - which is wholly contained within curve $D$. Similarly, in the range $k \geq 46$ the forced triads are in the range $16 \leq s_{F}<22$ - wholly contained within curve $E$. In the intermediate range $42 \leq k<46$ the forced triads are contained within both curves $D$ and $E$, where curve $E$ describes the net component energy transfer within the intermediate wavenumber modes of the nonlocal triadic interactions and curve $D$ describes the net energy transfer within the highest wavenumber modes of the nonlocal interactions, where all cancelations are taken into account. (Note that because $16 k_{F}=45.3$ is the wavenumber of the longest possible leg in a triad with $s=16$, curve $E$ must describe intermediate modes when $k<46$.) The observation that curve $E$ goes negative while $D$ rises to a positive maximum in all components $T_{\alpha \alpha}$ (Figs. 2 and 3 ) implies a strong forward cascading energy transfer from the intermediate to the highest wavenumber modes in highly nonlocal triads containing the low-wavenumber directly forced modes, where, by the triangle inequality, the intermediate and high wavenumber modes are close in scale. Although the precise location of the peaks is partly a consequence of the choice of $s$-bands, the existence of peaks of opposite sign in Figs. 2 and 3 , and similarly in figures 4 and 5 for the range $20 \leq k<40$, indicate a forward-cascading property within all directly forced nonlocal and marginally distant triadic interactions.

Consistent with Table I, the spanwise energy transfers in Fig. 3 when $k>46$ are dominated by forced triads with $16 \leq s<22$. At these wavenumbers all contributions from 
the directly forced triads are within curve $E$ irrespective of whether $\mathbf{k}$ is the intermediate or longest leg in individual triads. The highest $s$-band, $s \geq 32$, involves interactions with very low-energy modes at scales larger than the forced modes and, consequently, does not contribute significantly to energy transfer.

The differences between $T_{11}(k)$ and $T_{33}(k)$ in Figs. 2 and 3 for $k>46$ are, of course, directly related to anisotropy development at the small scales. Note that $T_{33}(k, s)$ (Fig. 3) exceeds $T_{11}(k, s)$ (Fig. 2) by nearly an order of magnitude at the higher wavenumbers, leading to a large gain in spanwise energy relative to $u_{1}$ and $u_{2}$ and strong anisotropy in the velocity components at the small scales. In fact, the nonlocal "forced" triads are so dominant in $T_{33}(k)$ that the less nonlocal interactions (for $s<8$ ) are essentially negligible, whereas the interactions with $2 \leq s<8$ do play a significant role in $T_{11}(k)$ (and $T_{22}(k)$ ).

\section{B. The apparent return to isotropy at the small scales $\left(t^{*}=3.83\right)$}

As the simulation progresses in time from $t^{*}=1.915$, energy from the forcing has cascaded from the large to small scales and the energy spectrum becomes less peaked (see Fig. 1). Consequently, the more local interactions not involving the forced modes gain in strength and compete with the forced distant interactions at high wavenumbers. $\mathrm{YBW}^{5}$ showed that the net effect of these nonforced local-to-nonlocal interactions is a reduction in anisotropy which progresses in cascading fashion from the large forced scales to smaller scales (in contrast with the development of small-scale anisotropy, which begins at the smallest scales and rapidly spreads in the direction of larger scales). YBW 5 also showed that, whereas the turbulence is still anisotropic at the small scales at $t^{*}=3.83$ (albeit, at a much reduced level), the structure of this anisotropy is such to make it undetectable by the spectral energy tensor. The small-scale anisotropy is apparent, however, with visualization of the energy distribution in Fourier-space, and through higher order moments in velocity.

The results just discussed for the time of maximum anisotropy are shown in equivalent form for $t^{*}=3.83$ in Table II, in Figs. $6-7$ for $40 \leq k<60$, and in Figs. 8-9 for $20 \leq k<40$. The highly nonlocal and distant interactions (fourth and fifth octaves) involving directly forced modes retain similar characteristics to those at $t^{*}=1.915$. Note that, like table I and Figs. 2-3, the nonlocal and marginally distant triadic interactions move more energy into the spanwise velocity component than the $u_{1}$ and $u_{2}$ components (compare, for example, curve $E$ in Fig. 6 with the same curve in Fig. 7 in the region $k>46$ ). Consequently, the nonlocal-to-distant triadic groups which continue to move the small scales away from an isotropic state.

However, there is an important difference between times $t^{*}=1.915$ and $t^{*}=3.83$ which is readily apparent by comparing Fig. $3\left(t^{*}=1.915\right)$ with Fig. $7\left(t^{*}=3.83\right)$ in the region $k>46$. Note that whereas in Fig. 3 the marginally distant group is, by far, the most important in transferring energy to the $u_{3}$ component, in Fig. 7 the marginally distant group (curve $E, s \sim 16-32$ ) competes with the "local-to-nonlocal" group (curves $B$ and $C, s \sim 2-8)$ in moving energy into $u_{3}$. Furthermore, Tạble II shows that the local-tononlocal interactions become important in all three components and tend to increase the energy in the $u_{1}$ and $u_{2}$ components relative to $u_{3}$; that is, the local-to-nonlocal groups 
move energy anisotropically in a manner opposite to the distant interactions (which favor spanwise energy). The combined effect is a large reduction of anisotropy in energy transfer and hence also the component energy spectra (Fig. 1).

It is important to appreciate that the reduction of anisotropy in component spectra is not achieved by a return to isotropy of the distant interactions which couple high wavenumber modes directly to the directly forced modes. Consistent with the analysis of YBW ${ }^{5}$, we find that the dominantly forward cascading local-to-nonlocal interactions tend to move the small scales towards isotropy. However, due to the continuing anisotropy at the forced scales, the small scales retain a finite, though much reduced, level of small-scale anisotropy through the dynamics of the distant triadic group.

\section{Net energy transfer vs. anisotropy at the small scales}

Having taken all cancelations within the different triadic groups into account, we have arrived at the same conclusions as $\mathrm{YB}^{4}$ and $\mathrm{YBW}^{5}$ - the marginally distant triadic group which does not entirely cancel out, is responsible for the initial development of anisotropy at the small scales, and is responsible for the maintenance of anisotropy at the small scales at later times. However, due to the large amount of energy deposited into the large scales by the forcing, and because of the limited separation in scales, we have also shown the existence of direct energy transfer between the forced modes and the small scales. The results of Zhou ${ }^{7}$ and Brasseur \& $\mathrm{Wei}^{11}$ indicate that, in the absence of forcing, forward-cascading energy transfer is primarily within local-to-nonlocal triadic interactions with scale separations up to roughly 10 . Zhou ${ }^{7}$ showed that, in the inertial range of isotropic simulations, the strongest contributions to net energy flux are within scale separations of roughly 1.8 to 5 , or so. Consequently, for the distant triadic group to influence the structure of the smallest scales in high Reynolds number turbulence, it is necessary to show that the influence of the distant triadic group on small-scale structure remains intact while the direct transfer of energy from the large to the small scales diminishes.

Consider the high-wavenumber shell $k=50$, separated in scale from the forced modes by a factor of about 18 , and influenced by marginally distant triads ${ }^{11}$. In Figs. 10 (a) and (b) we plot the net energy transfer $T(k, s)$ into, or out of, shell $k=50$ as a function only of scale separation $s$ at the time of peak anisotropy $\left(t^{*}=1.915\right)$ and at the later time of diminished anisotropy $\left(t^{*}=3.83\right)$. To improve resolution, $s$ is separated into half octave bands $\left(2^{N / 2}, N=0,12\right)$. The only $s$-band which contains the forced triads is shown cross-hatched. Note that except for this $s$-band, net energy transfer is into shell $k$ and is dominated by local-to-nonlocal interactions up to roughly 10-15 (consistent with Brasseur $\& \mathrm{Wei}^{11}$ ) and with the strongest contribution from nonlocal triadic interactions with scale separations roughly 2 to 8 (consistent with $\mathrm{Zhou}^{7}$ ). The spike in Fig. 10(a) indicates that, whereas a great deal of energy flows directly from the forced modes to shell $k$ at the time of peak anisotropy, at the later time (Fig. 10(b)) the forced triadic group contributes a relatively small proportion of the net energy flux into the high wavenumber shell.

In contrast with Figs. 10 (a)-(b), consider Figs. 11(a) and (b) which show the anisotropy in component energy transfer as a function of scale separation $s$ for shell $k=50$ at the same two times as in Figs. 10 (a)-(b). Anisotropy in component energy transfer is quantified 
by the ratio of maximum to minimum component energy transfer, in absolute value, in given $s$-band of triadic interactions (the ratio is 1 in isotropic turbulence). Like Figs. 10 (a)-(b), the marginally distant $s$-band containing the forced triads is shown cross-hatched. Because of the forcing, this band stands out as spikes in the anisotropy of component energy transfer in shell $k$. Except for these spikes, note the increase in anisotropy which begins at scale separation $s \approx 10$, then rapidly increases with increasing scale separation $s$. It is interesting that the increase in anisotropy begins at roughly the same scale separation that the dominance of cascading local-to-nonlocal triads in $T(k, s)$ ends. We conclude that the anisotropizing influences within the large scales are most strongly felt within the distant triadic group, and the more distant the triadic group, the stronger is the anisotropizing influence of that group. Note that cancelation within the distant triads are taken into account in this result.

Figures 11 (a)-(b) have also uncovered a surprising and curious result - the most local triadic groups, those where $s_{\sim}^{<2}$, appear to have a stronger anisotropizing influence than the local-to-nonlocal triadic group, $2_{\sim}^{<} s_{\sim}^{<} 10$. We do not have an definitive explanation for this unexpected behavior. Perhaps it is a consequence of a dominantly inverse-cascading characteristic that has been observed within the most local triadic interactions in the calculations by Brasseur \& Wei ${ }^{11}$, or perhaps it is simply a numerical artifact of the low levels of component energy transfer in the most local triadic interactions (see tables I and II).

Figures 10 and 11 have shown that without forcing, the most distant interactions do not significantly contribute to net energy transfer; yet it is these interactions that dominate the anisotropic redistribution of component energy when the large scales are nonisotropic. As might be expected, forcing enhances the anisotropizing influence of the marginally distant distant group within the forced triads. For this long-range effect to persist to higher Reynolds numbers, net energy transfer within the forced triads should decrease with increasing scale separation $s$ while the anisotropy in component energy transfer should increase with $s$. This is shown to be the case in Figs. 12 and 13.

In Fig. 12 we plot $T(k, s)$ against $s$ only for the $s_{F}$-bands that contain forced triads, using Eq. 7 for each $k$ shell. Consequently, each $s$-band plotted is within a different $k$ shell and because the $k$ shells are narrow (thickness $\Delta k=1$ ), so are the $s_{F}$ bands which are plotted in the figure. Note that, whereas the magnitude of $T(k, s)$ is higher at the later time due to the large increase in energy from the initiation of forcing, at both $t^{*}=1.915$ and $t^{*}=3.83$ the net transfer within the forced triadic groups rapidly decreases with increasing scale separation, approaching zero asymptotically at large $s$.

In Fig. 13 we plot the same measure of anisotropy as in Figs. 10 and 11, but only for the forced $s$-bands, $s_{F}$, as in Fig. 12. The earlier time is shown with solid lines and the later time with lashed lines. Because the $s_{F}$-bands are narrow, the variation of the ratio with $s_{F}$ is very noisy, particularly at the later time when the influence of the distant triadic group has weakened substantially. Nevertheless, it is apparent from these figures that in contrast with net energy transfer $T(k, s)$ which decreases rapidly, the anisotropy in component energy transfer increases overall with increasing $s_{F}$, indicating that the anisotropizing influence of the forced triads remains strong within the distant triadic group as scale separation $s$ increases. Furthermore, although the net level of anisotropy in component energy transfer decreases from the time of peak anisotropy, the anisotropizing influence of the distant group is still strong at later times. These results suggest that the anisotropizing influence of the 
distant triadic group does not die at higher scale separations (suggesting higher Reynolds numbers), even as the scale separation becomes sufficiently high to block direct energy transfer between the forced large scales and the smallest dynamic scales of motion.

\section{Discussion: cancelation within the distant triadic group}

Waleffe ${ }^{12}$ has argued that when all triadic interactions are summed together, strong mutual cancelation between the positive and negative nonlocal interactions (called " $R$ " type contributions therein) would result, leaving no effective influence of the distant triadic group on the anisotropic development of small scale structure and, therefore, on local isotropy. The results above have shown that the marginally distant group does not completely cancel out and is responsible for the development of small-scale anisotropy in the forced simulations. Furthermore, the long-range interactions remain an influence on small-scale structure at later times when the effects of forcing have weakened substantially and the small scale motions evolve within a sea of competing local, nonlocal and distant triadic interactions.

To show the net effects of cancelation more completely, the cumulative contributions of the dominant nonlocal classes of $T(k, s)$ are shown for all three velocity components in Figs. 14 and 15 at times $t^{*}=1.915$ and $t^{*}=3.83$, respectively, for the range $40 \leq k<60$. Specifically, the complete component energy transfers $T_{\alpha \alpha}(k)$ (closed symbols) are compared with the net contributions from triads with scale separation $s \sim 8-32$ (open symbols). Note that whereas the complete energy transfer spectrum strongly favors the $z$-component at $t^{*}=1.915$, at the later time $t^{*}=3.85$ the complete energy transfer is isotropically distributed within components. The nonlocal-to-distant triadic interactions, on the other hand, redistribute energy anisotropically at both times, providing $u_{3}$ with more energy than $u_{1}$ and $u_{2}$ at the small scales.

These results, explicitly taking all cancelations into account, are consistent with those of $\mathrm{YBW}^{5}$, showing that the highly nonlocal and marginally distant interactions do not cancel out. This issue was argued previously by $\mathrm{YBW}^{5}$ based on the observation that the small scales did, in fact, become anisotropic in some detail according to an analysis of the triadic equations in the limit of asymptotically distant interactions.

\section{Conclusions}

The development of small-scale anisotropy in response to large-scale forcing previously reported by $\mathrm{YB}^{4}$ and $\mathrm{YBW}^{5}$ has been studied by an analysis of triadic energy transfer using the $T(k, s)$ formalism introduced by Zhou ${ }^{6,7}$. Comparison of triadic interactions at different values of the scale disparity parameter $s$ confirms earlier interpretations that the anisotropy development is a result of highly nonlocal or marginally distant interactions that couple high wavenumber modes with a low-wavenumber directly forced mode ${ }^{4-5}$. In continued forcing, the distant interactions remain active and anisotropic even as the cascading effects of more local interactions competes strongly with the long-range effects, resulting in an overall partial reduction in anisotropy of the component energy spectra at later times, 
confirming the analysis if $\mathrm{YBW}^{5}$ which did not directly analyze the extent of cancelation within nonlocal and distant triadic groups. This study demonstrates that the nonlocal-todistant interactions do not cancel out under summation as argued by Waleffe ${ }^{12}$.

We also find that the effects of forcing result in net forward energy transfer within the nonlocal-to-distant triadic group. Because our analysis is at low Reynolds numbers with a rapid decrease in spectral energy at high $k$, this last observation is not inconsistent with Waleffe's ${ }^{15}$ later result that net forward cascade exists in the distant triadic group if the spectrum falls off faster than $k^{7 / 3}$.

Additional analysis of net energy transfer versus net anisotropy in energy transfer within a high wavenumber shell $k$ has shown that, whereas the forward cascading effects become progressively more confined to local-to-nonlocal scale interactions with scale separations less than roughly 10 (consistent with Zhou ${ }^{7}$ and Brasseur \& Wej ${ }^{11}$ ), the anisotropizing influence are most strongly within the distant triadic group and increase with scale separation $s$, consistent with the arguments of Brasseur \& Wei ${ }^{11}$. Most importantly, we have shown that direct energy transfer from the forced modes to higher wavenumber modes decreases with scale separation while, at the same time, the net anisotropizing influences of the forced distant group increases with scale separation $s$. These results support the argument that the anisotropizing influence of coherent anisotropic large-scale forcing on the smallest dynamical scales through direct long-range interactions should persist to higher Reynolds numbers. 


\section{LargeReferences}

1. A.N. Kolmogorov, "The local structure of turbulence in incompressible viscous fluid for very large Reynolds number," Dokl. Akad. Nauk SSSR, 30, 301 (1941)

2. G.K. Batchelor, The Theory of Ilomogeneous Turbulence, Cambridge University Press, Cambridge, England (Sec. 6.3, for example), (1953).

3. J. A. Domaradzki and R.S. Rogallo, "Local energy transfer and nonlocal interactions in homogeneous, isotropic turbulence", Phys. Fluids A, 2, 413 (1990).

4. P.K. Yeung and J. G. Brasseur, "The response of isotropic turbulence to isotropic and anisotropic forcing at large scales," Phys. Fluids A, 3, 884 (1991).

5. P.K. Yeung, J. G. Brasseur, and Q. Wang, "Dynamics of direct large-small scale couplings in coherently forced turbulence: Concurrent physical and Fourier-space view", To appear, J. Fluid Mech., (1994)

6. Y. Zhou, "Degrees of locality of energy transfer in the inertial range", Phys. Fluids $A, 5,1092$ (1993)

7. Y. Zhou, "Interacting scales and energy transfer in isotropic turbulence", Phys. Fluids $A, 5,2511$ (1993)

8. K. Ohkitani and S. Kida, "Triad interactions in a forced turbulence", Phys. Fluids $A, 4,794$ (1992).

9. J.A. Domaradzki, "Nonlocal triadic interactions and the dissipation range of isotropic turbulence," Phys. Fluids A, 4, 2037 (1992).

10. S. Kida, R.H. Kraichnan, R.S. Rogallo, F. Waleffe, and Y. Zhou, "Triad interactions in the dissipation range" in Proc. of the Summer Program 1992, Center for Turbulence Research, Stanford Univ. and NASA AMES Research Center.

11. J.G. Brasseur and C.-H. Wei, "Interscale dynamics and local isotropy in high Reynolds number turbulence within triadic interactions", Phys. Fluids, 6, 842 (1994).

12. F. Waleffe, "The nature of triad interactions in homogeneous turbulence", Phys. Fluids $A, 4,350(1992)$

13. R.H. Kraichnan, "The structure of isotropic turbulence at very high Reynolds numbers," J. Fluid Mech., 5, 497 (1959)

14. R.H. Kraichnan, "Inertial-range transfer in two- and three-dimensional turbulence," J. Fluid Mech., 47, 525 (1971)

15. F. Waleffe "Inertial transfers in the helical decomposition," Phys. Fluids A, 5, 677 (1993). 
Table I: Component energy transfer by different scale disparities, at $t^{*}=1.915$ (values in $10^{-7}$ )

\begin{tabular}{|c|c|c|c|c|c|c|c|}
\hline & $s: 1-2$ & $2-4$ & $4-8$ & $8-16$ & $16-32$ & $\geq 32$ & Total \\
\hline$k=22$ & \multicolumn{3}{|c|}{$7.6 \leq s_{F} \leq 8.95$} & & & & \\
\hline$T_{11}(k, s)$ & 13.38 & 48.02 & 167.07 & -116.16 & -2.58 & 0 . & 109.73 \\
\hline$T_{22}(k, s)$ & 10.31 & 36.55 & 159.68 & -109.66 & -2.13 & 0 . & 94.75 \\
\hline$T_{333}(k, s)$ & -5.92 & -22.39 & 862.50 & -614.44 & -1.11 & 0 . & 218.64 \\
\hline$T(k, s)$ & 8.89 & 31.09 & 593.13 & 420.13 & -1.75 & 0 . & \\
\hline$k=30$ & \multicolumn{3}{|c|}{$10.43 \leq s_{F} \leq 11.78$} & & & & \\
\hline$T_{11}(k, s)$ & 1.64 & 11.30 & 7.11 & 9.46 & 0.22 & 0 . & 29.7 \\
\hline$T_{22}(k, s)$ & 1.75 & 10.18 & 5.95 & 9.29 & 0.04 & $\theta$. & 27.2 \\
\hline$T_{333}(k, s)$ & -0.42 & 2.38 & 5.46 & 77.74 & 0.40 & 0 . & 85.2 \\
\hline$T(k, s)$ & 1.49 & 11.93 & 9.26 & 48.25 & 0.33 & 0 . & \\
\hline$k=44$ & \multicolumn{3}{|c|}{$15.38 \leq s_{F} \leq 16.73$} & & & & \\
\hline$T_{11}(k, s)$ & 0.06 & 0.82 & 0.94 & 3.90 & -2.70 & 0 . & 3.02 \\
\hline$T_{22}(k, s)$ & 0.07 & 0.79 & 0.80 & 3.56 & -2.44 & 0 & 2.78 \\
\hline$T_{3: 3}(k, s)$ & 0.00 & 1.02 & 1.57 & 27.73 & -18.40 & -0.02 & 11.9 \\
\hline$T(k, s)$ & 0.07 & 1.38 & 1.66 & 17.6 & -11.77 & -0.01 & \\
\hline$k=.50$ & \multicolumn{3}{|c|}{$17.5 \leq s_{F} \leq 18.85$} & & & & \\
\hline$T_{11}(k, s)$ & 0.02 & 0.31 & 0.41 & 0.16 & 0.32 & 0 . & 1.22 \\
\hline$T_{22}(k, s)$ & 0.02 & 0.29 & 0.34 & 0.14 & 0.29 & 0 . & 1.08 \\
\hline$T_{333}(k, s)$ & 0.00 & 0.49 & 0.72 & 0.51 & 2.88 & 0.02 & 4.62 \\
\hline$T(k, s)$ & 0.02 & 0.55 & 0.74 & 0.41 & 1.75 & 0.01 & \\
\hline
\end{tabular}


Table II: Component energy transfer by different scale disparities, at $t^{*}=3.83$

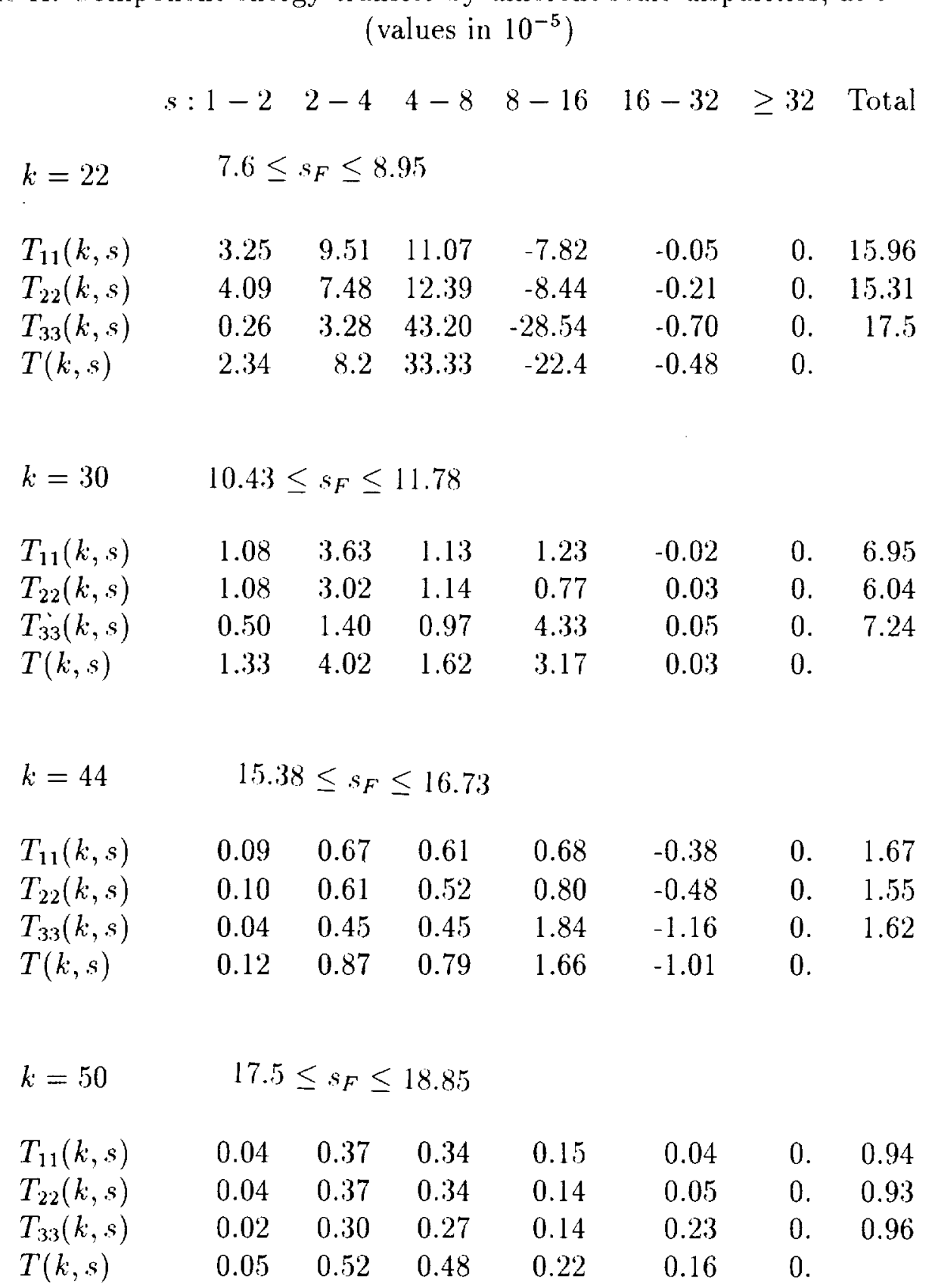

Note: Data values less than $10^{-7}$ in magnitude are shown as zeros. 


\section{Figure captions}

Fig. 1: Component, energy spectra at beginning of forcing, $t^{*}=0$ (ummarked lines), time of maximal anisotropy, $t^{*}=1.915$ (open symbols), and reduced anisotropy at later time, $l^{*}=3.83$ (closed symbols). $T_{11}(k), T_{22}(k)$ and $T_{333}(k)$ are indicated by triangles, circles, and squares respectively.

Fig. 2: $T_{11}(k, s)$ vs $k$ in the range $40 \leq k<60$, for different scale disparity parameters $(s)$, at $t^{*}=1.915$. Lines $A-F$ are for $s$ in the octaves $2^{n} \leq s<2^{n+1}, n=$ $0,1,2,3,4,5$., respectively (as in Tables 1 and II)

Fig. 3: Same as Fig. 2, lunt for $T_{33}(k)$.

Fig. 4: Same as Fig. 2, but for $k$ in the range $20 \leq k<40$.

Fig. 5: Same as Fig. 4, but for $T_{33}(k)$.

Fig. 6: Same as Fig. 2, but at $t^{*}=3.83$.

Fig. 7: Sime as Fig. 3, hut at $t^{*}=3.83$.

Fig. 8: Same as Fig. 4 , but at $t^{*}=3.83$.

Fig. 9: Same as Fig. 5, but at $t^{*}=3.83$.

Fig. 10: Net energy transfer $T(k, s)$ in shell $k=50$ within different triadic groups with scale disparity $s$ in half-octaves, (a) at the time of peak small scale anisotropy $\left(t^{*}=1.915\right)$ and $(b)$ at the later time $t^{*}=3.83$. The only $s$-band which contains the forced triads is shown cross-hatched.

Fig. 11: Anisotropy in component energy transfer, $\left|T_{\alpha \alpha}\right|_{\text {max }} /\left|T_{\alpha \alpha}\right|_{\text {min }}$, in shell $k=50$ within different triadic groups with scale disparity $s,(a)$ at the time of peak small scale anisotropy $\left(t^{*}=1.915\right)$ and $(b)$ at the later time $t^{*}=3.83$. The only s-band which contains the forced triads is shown cross-hatched.

Fig. 12: Net energy transfer $T(k, s)$ within the forced triadic groups as a function of scale disparity $s_{F}$, at the time of peak small scale anisotropy, $t^{*}=1.915$ (solid line), and at the later time $t^{*}=3.83$ (dashed line).

Fig. 13: Same as Fig. 12, but for anisotropy in component energy transfer, $\left|T_{\alpha \alpha}\right|_{\text {max }} /\left|T_{\alpha \alpha}\right|_{\text {min }}$.

Fig. 14: ('omponent transfers $T_{11}(k, s)$ (triangles), $T_{22}(k, s)$ (circles), $T_{33}(k, s)$ (squares), summed $8 \leq s<32$ (open symbols) in the range $40 \leq k<60$, and 
compared with the total transfers $\left(T_{11}(k), T_{22}(k), T_{33}(k)\right)$ (closed symbols) at $t^{*}=$ 1.915 .

Fig. 15: Same as Fig. 14, but at $t^{*}=3.83$. 
Fig. 1

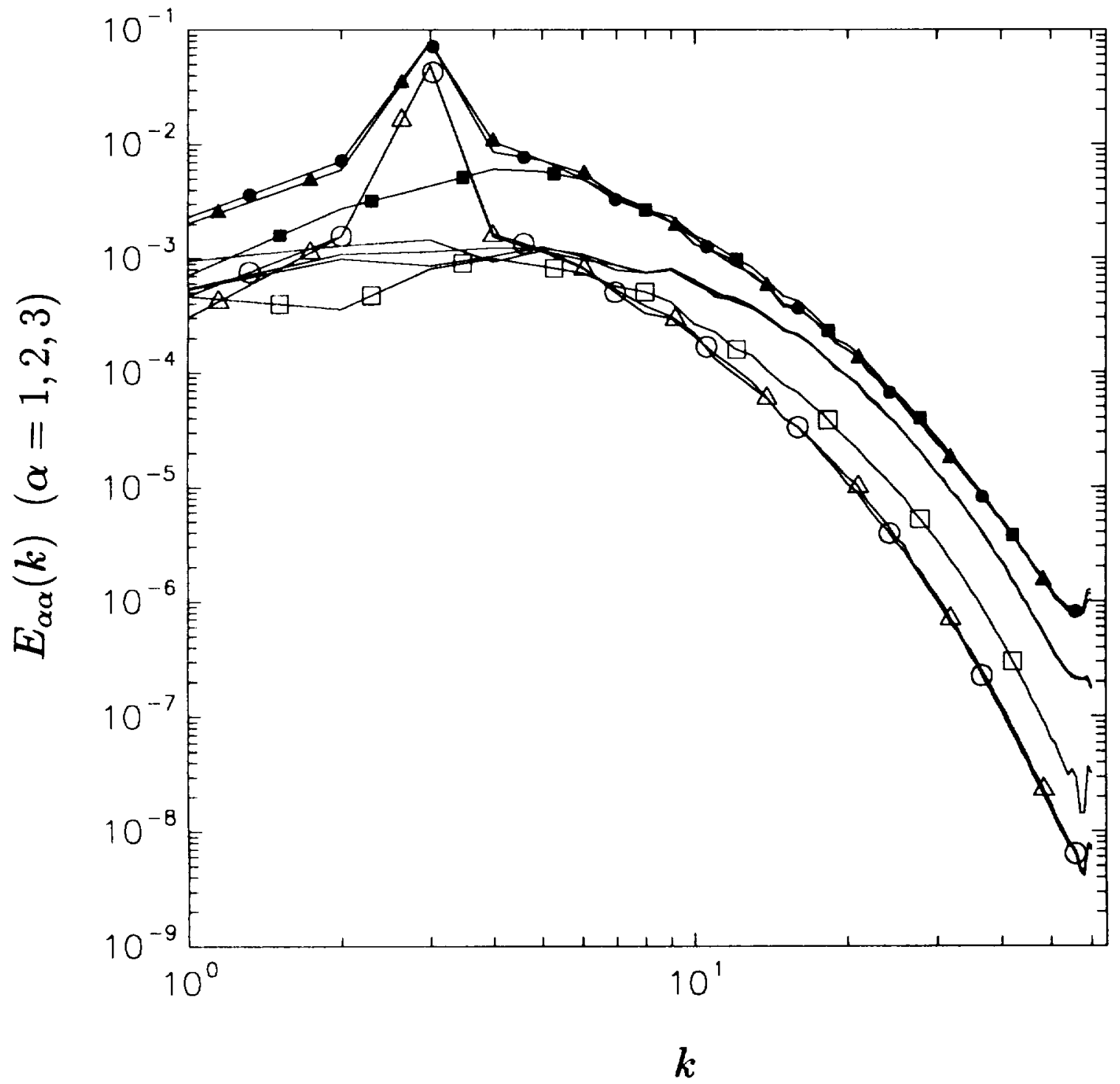


Fig. 2

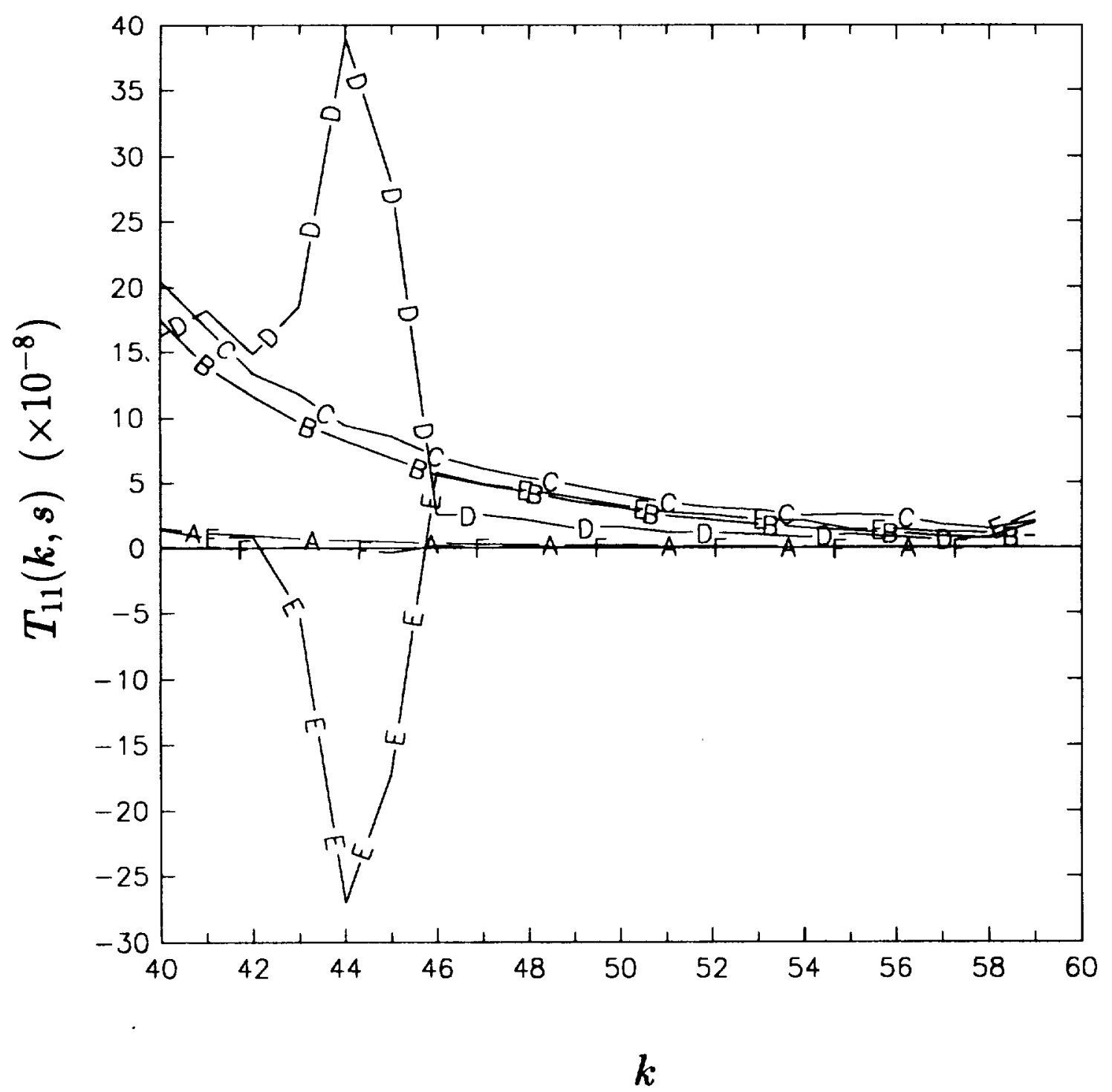


Fig. 3

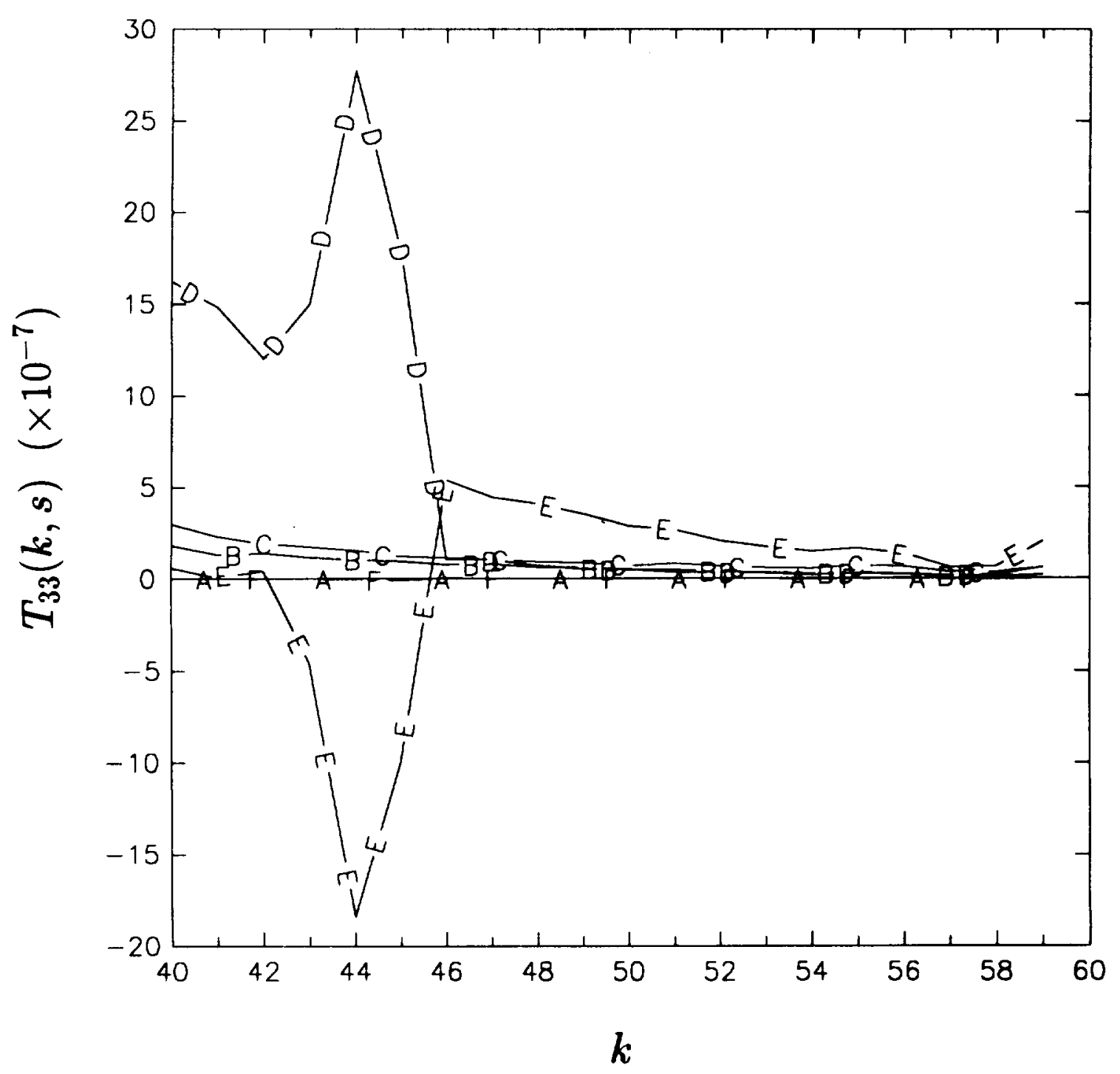


Fig. 4

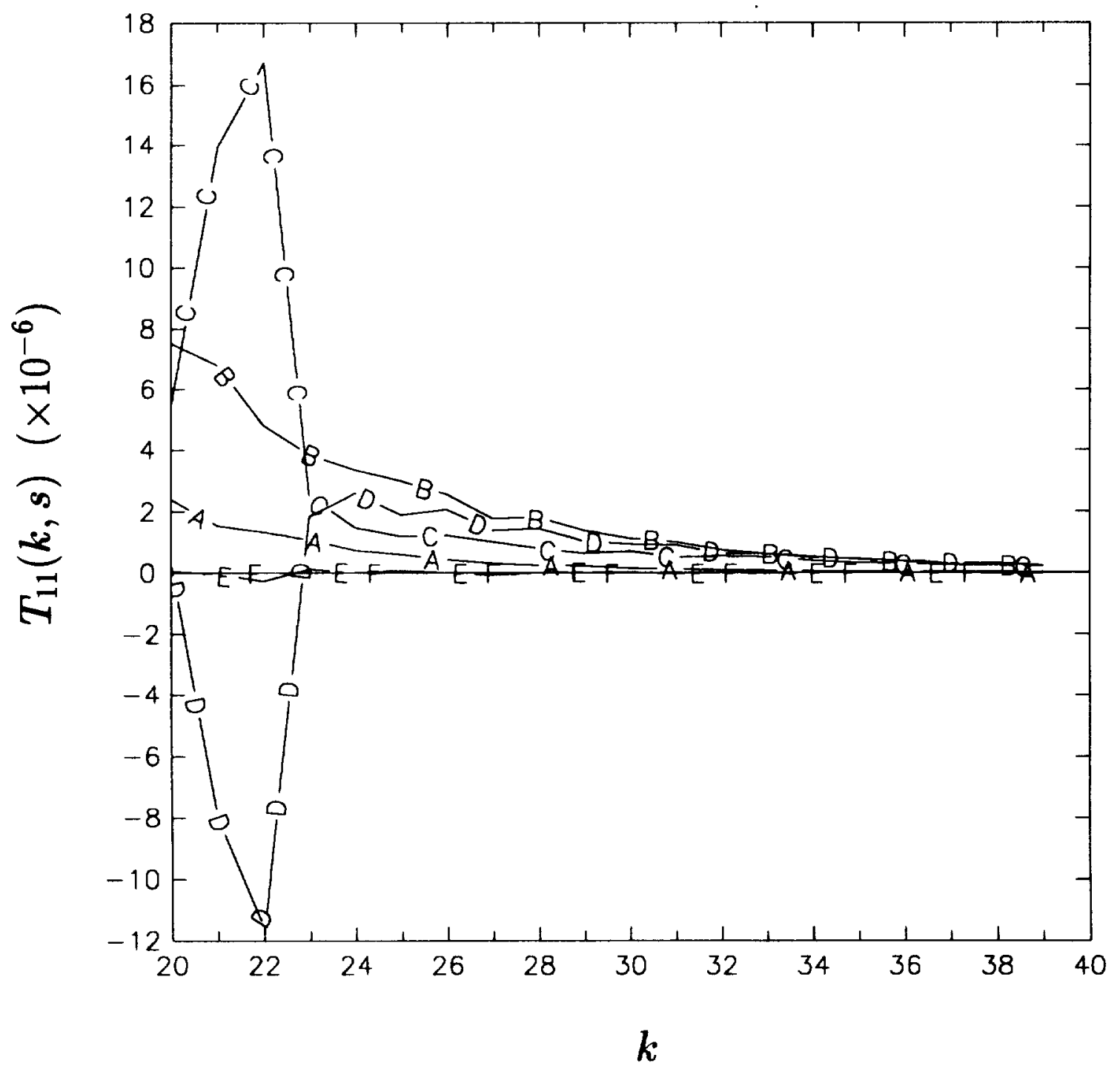


Fig. 5

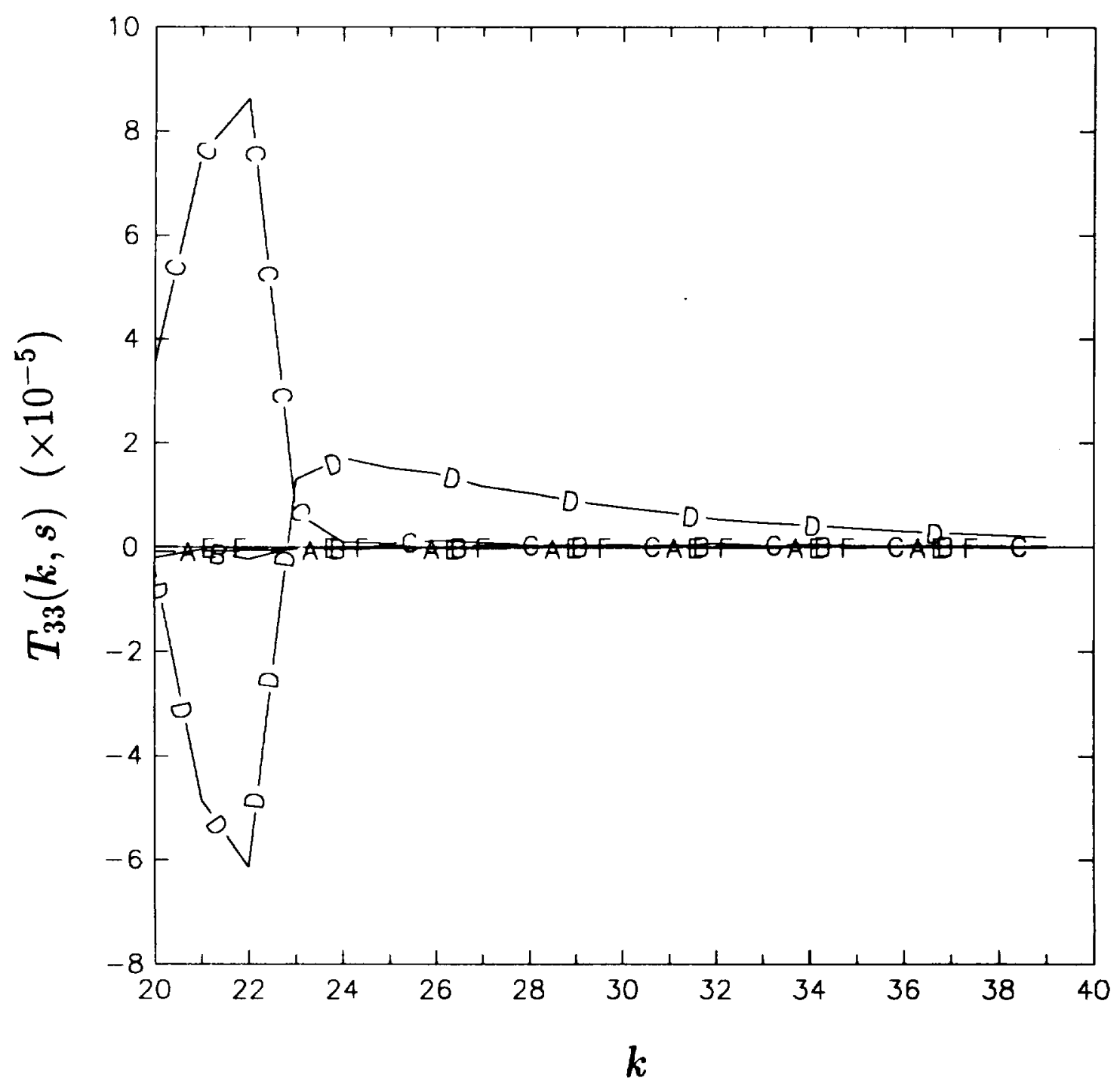


Fig. 6

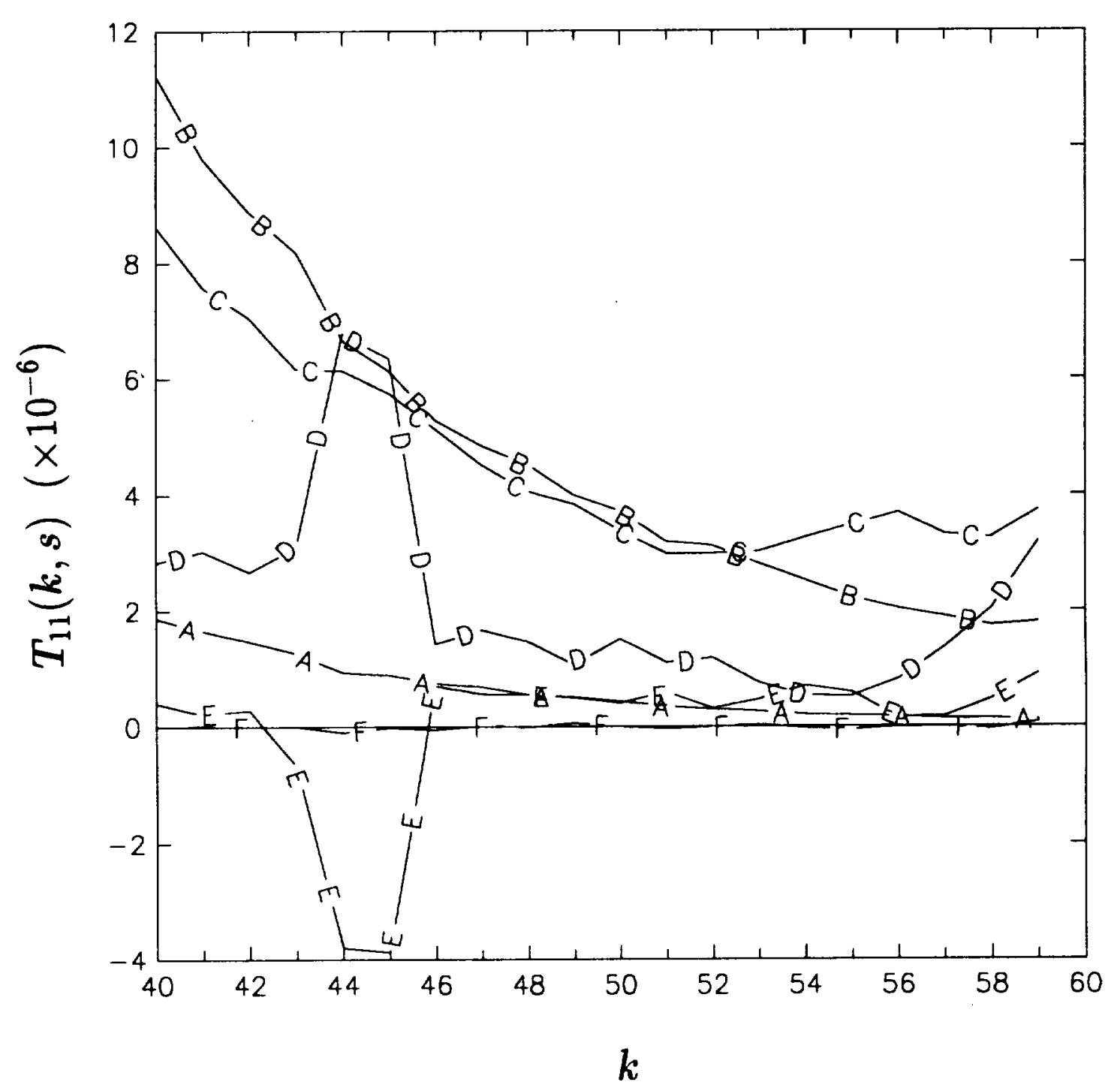


Fig. 7

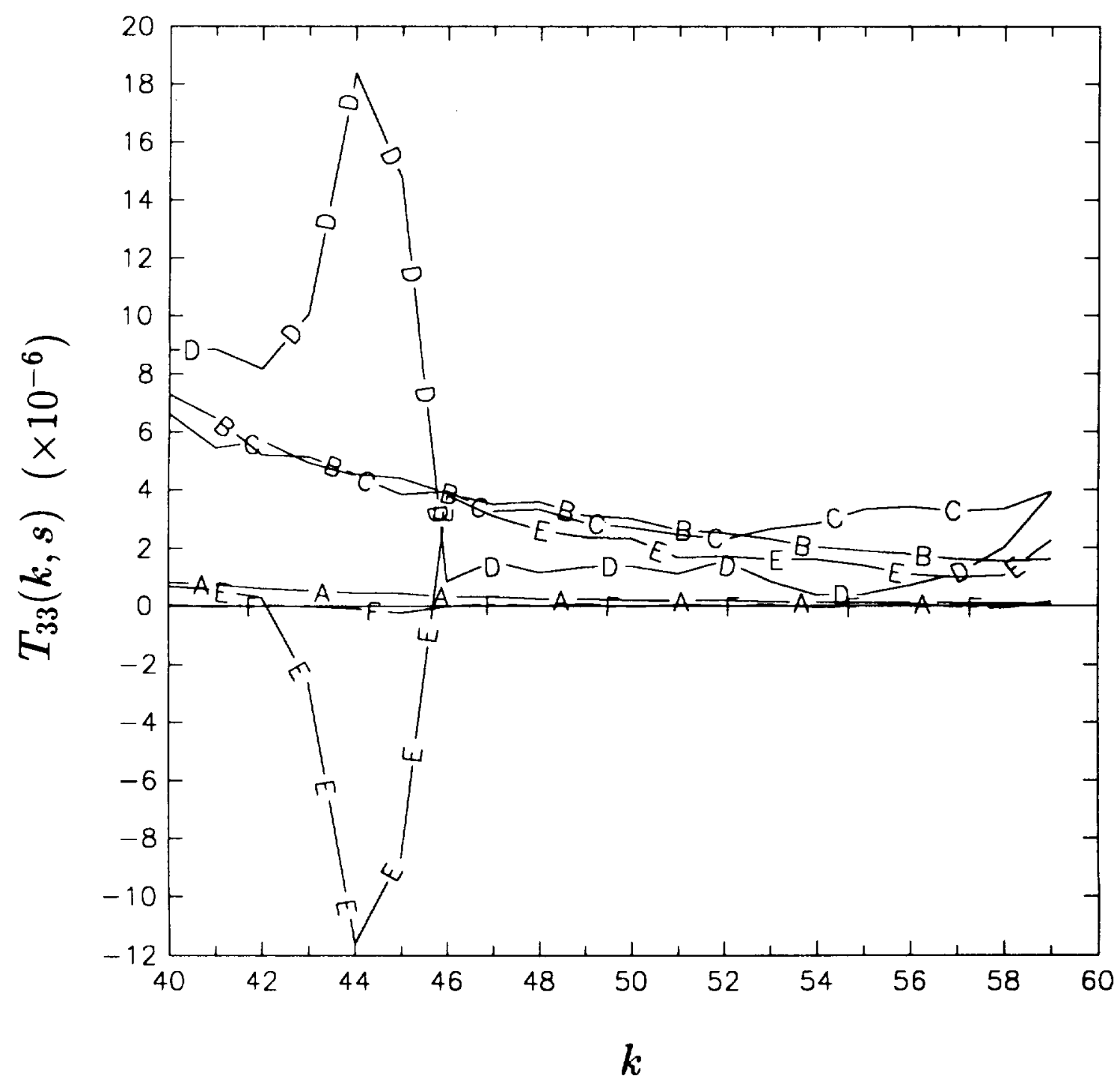


Fig. 8

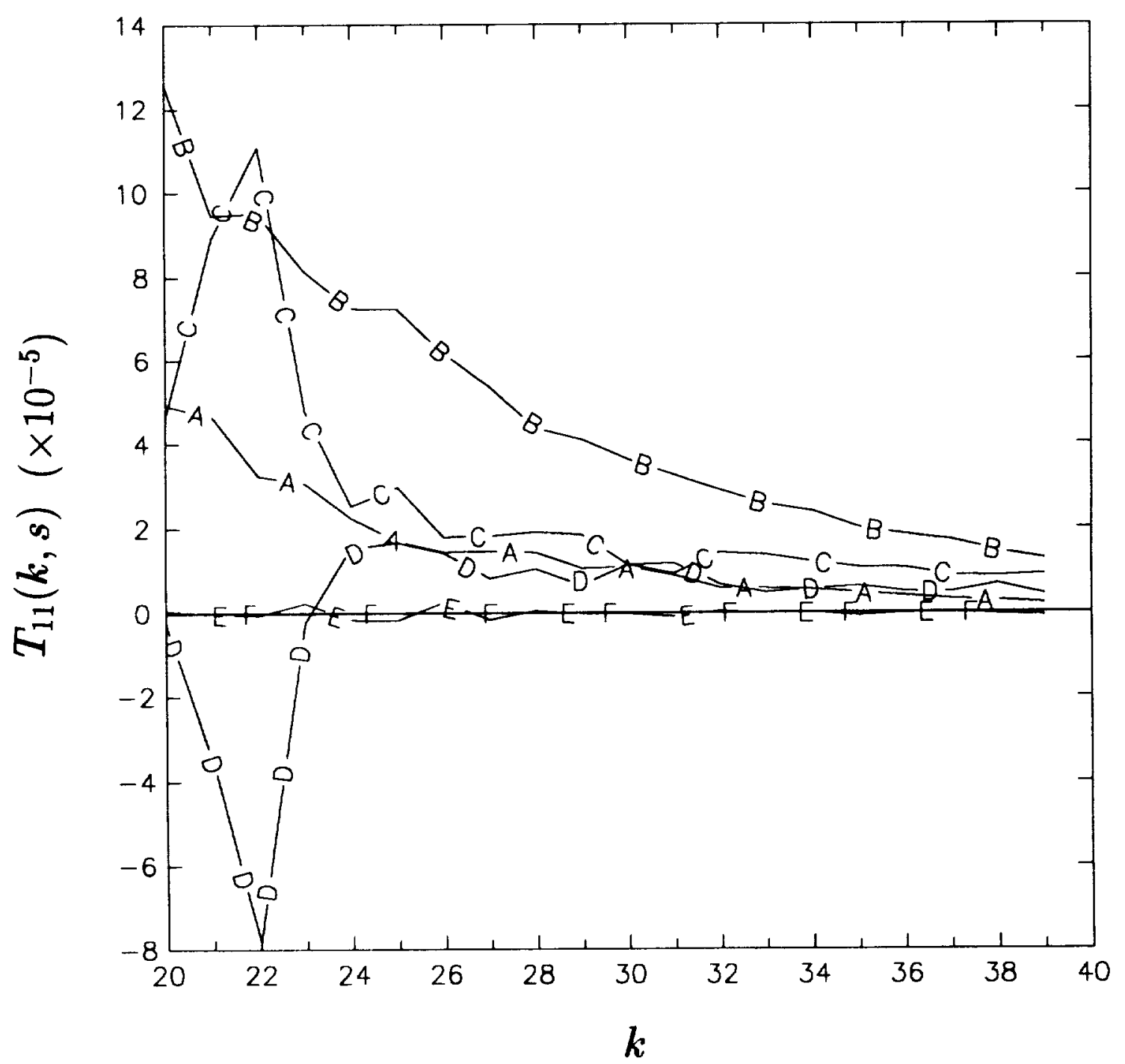


Fig. 9

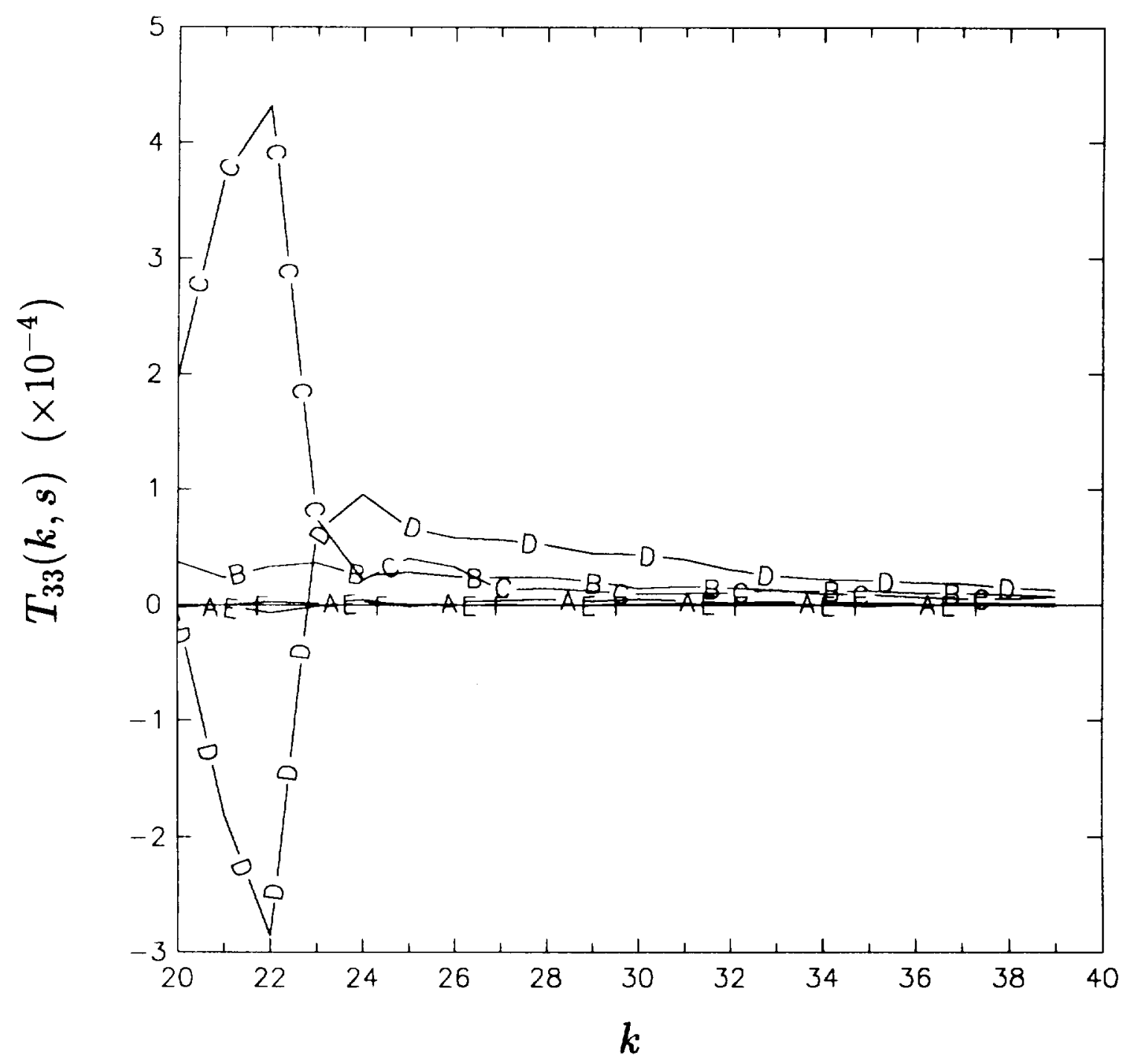


Fig. 10(b)

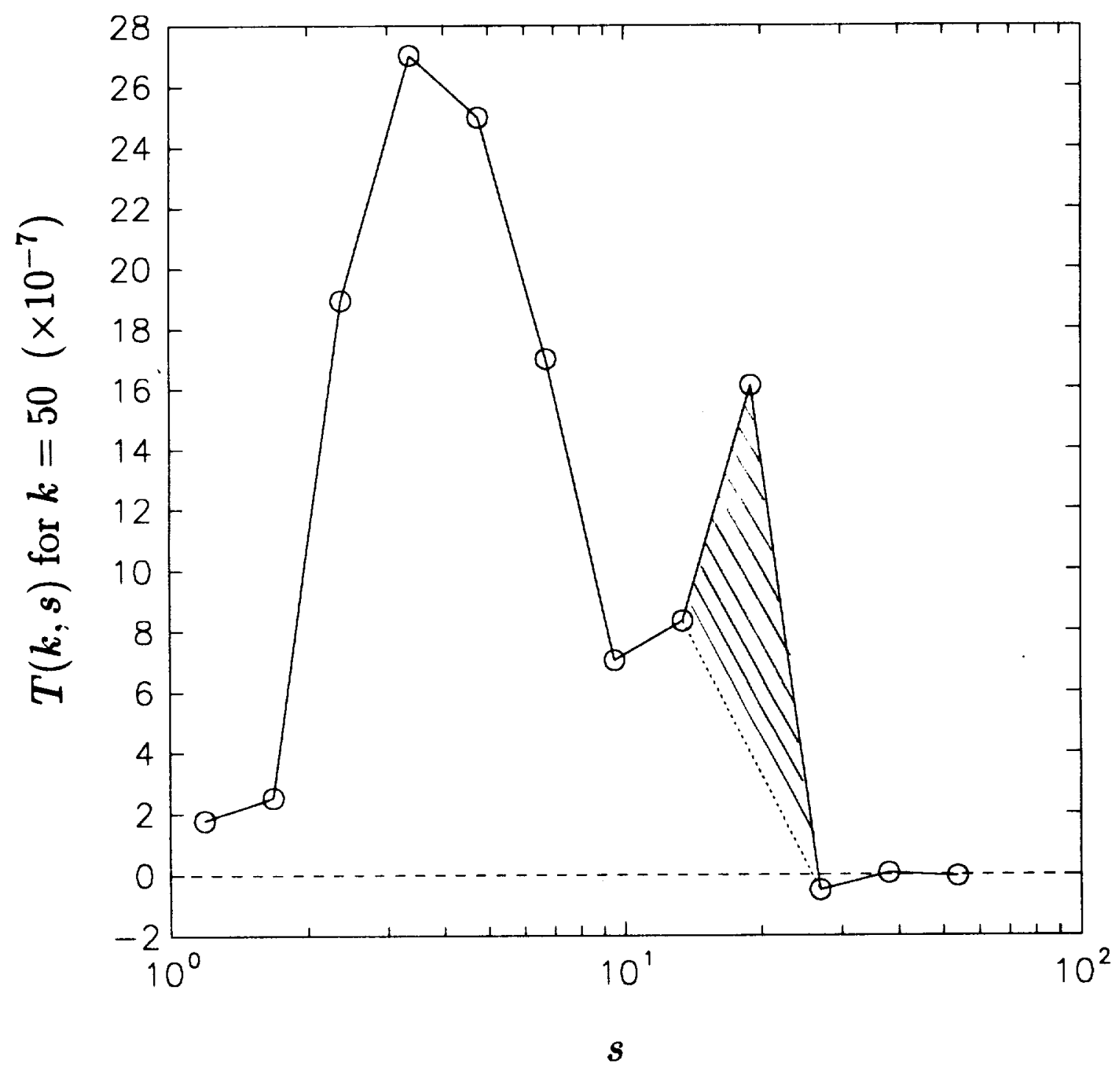


Fig. 10(a)

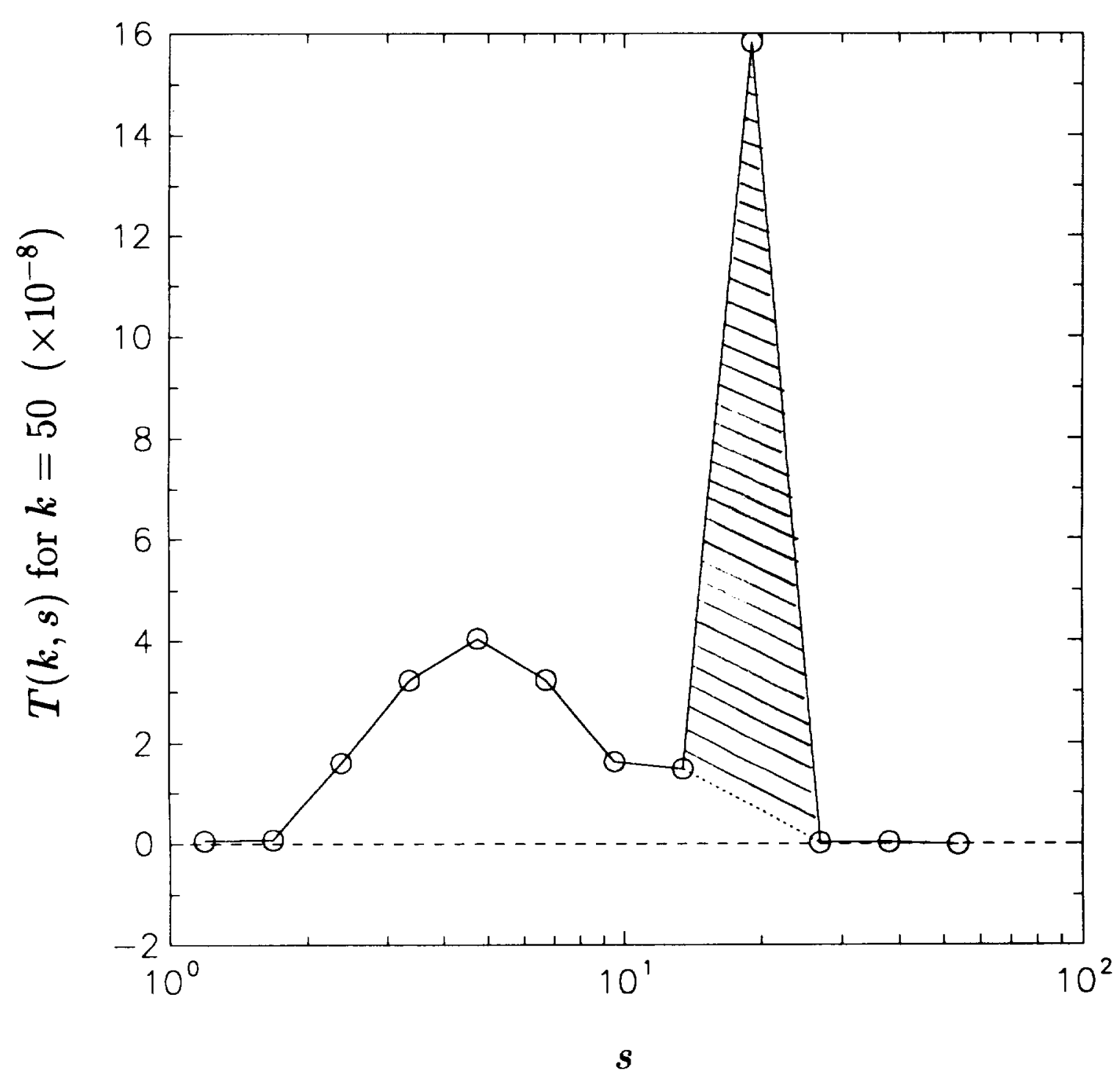


Fig. 11(a)

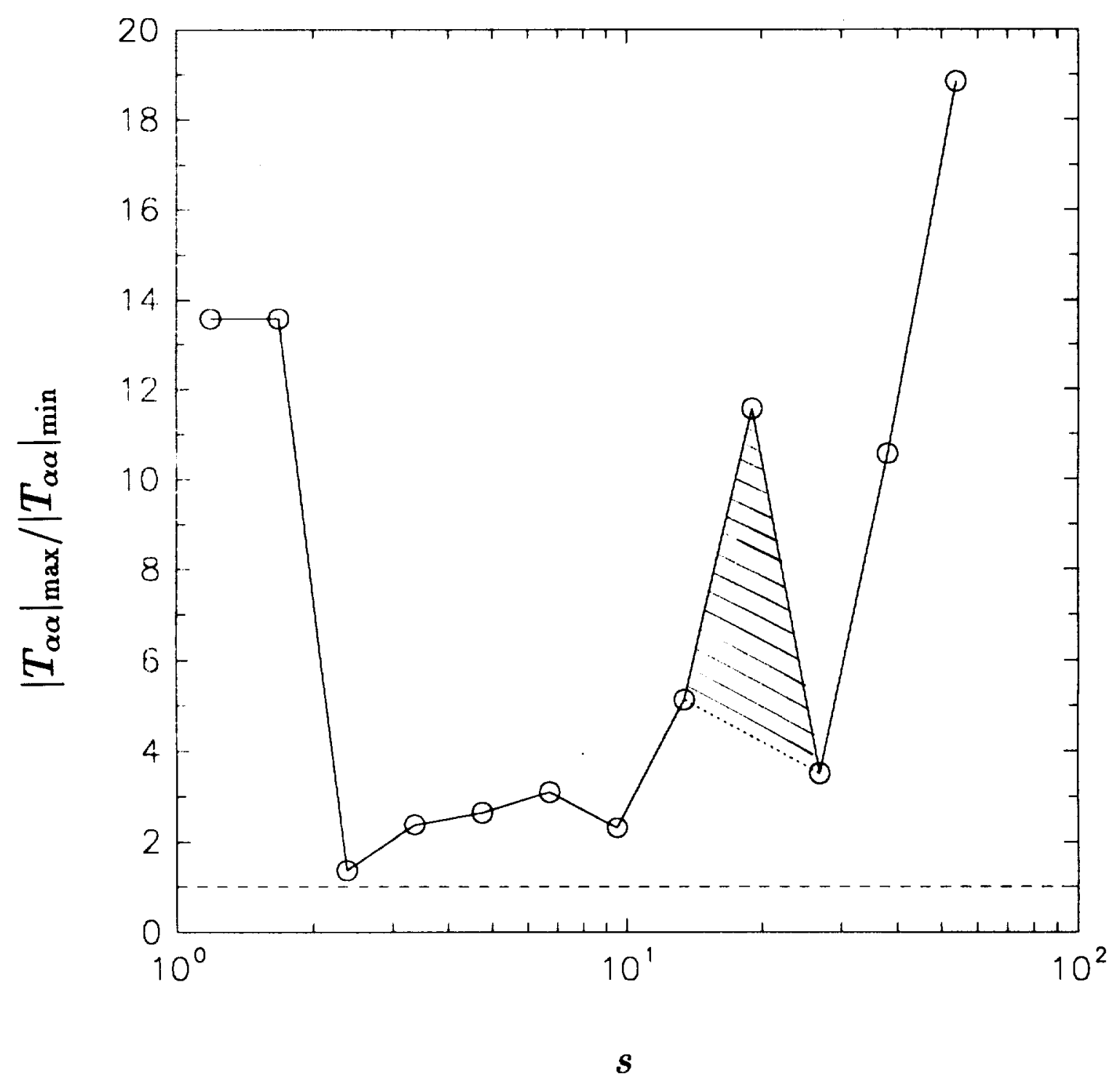


Fig. 11(b)

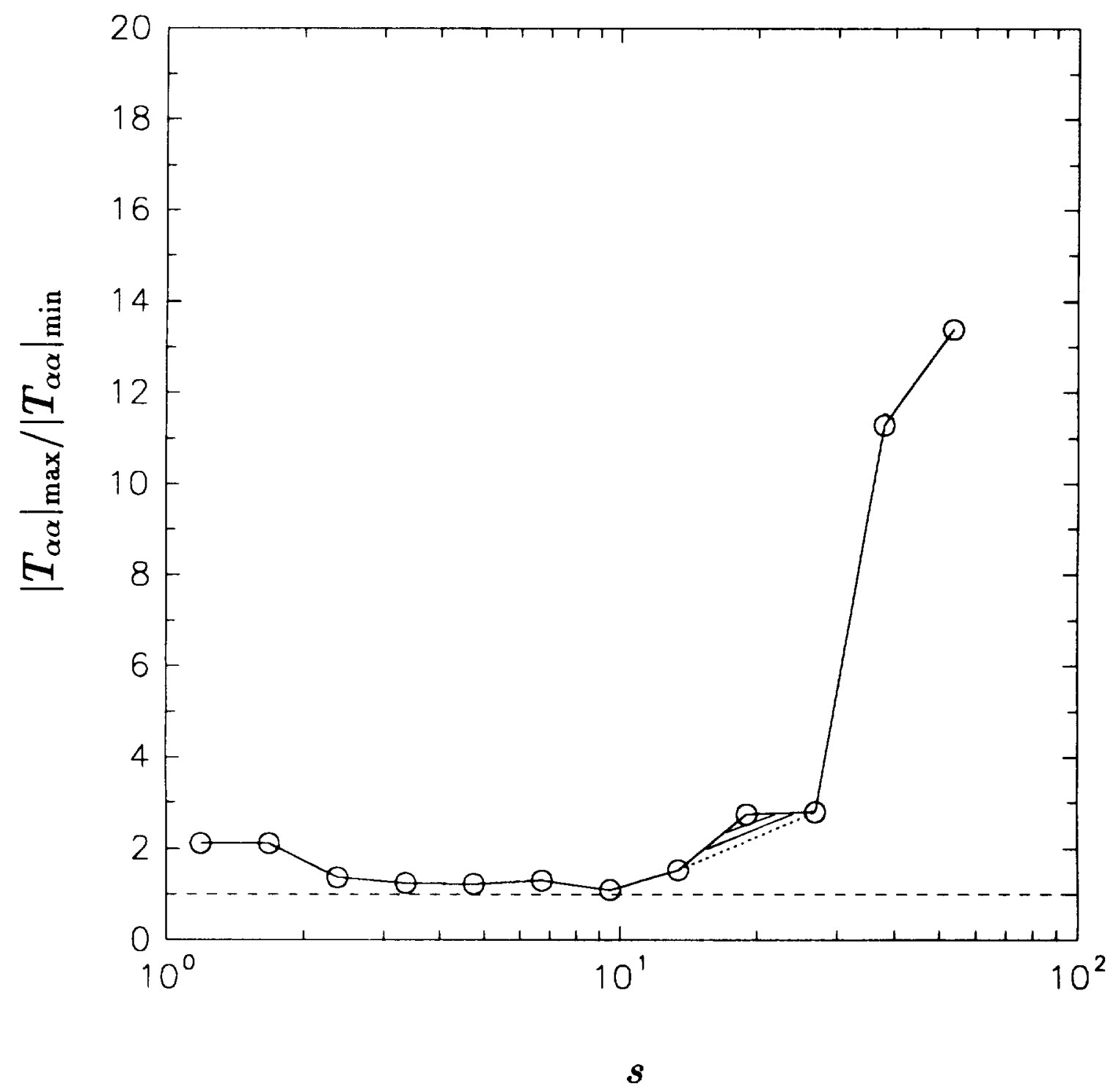


Fig. 12

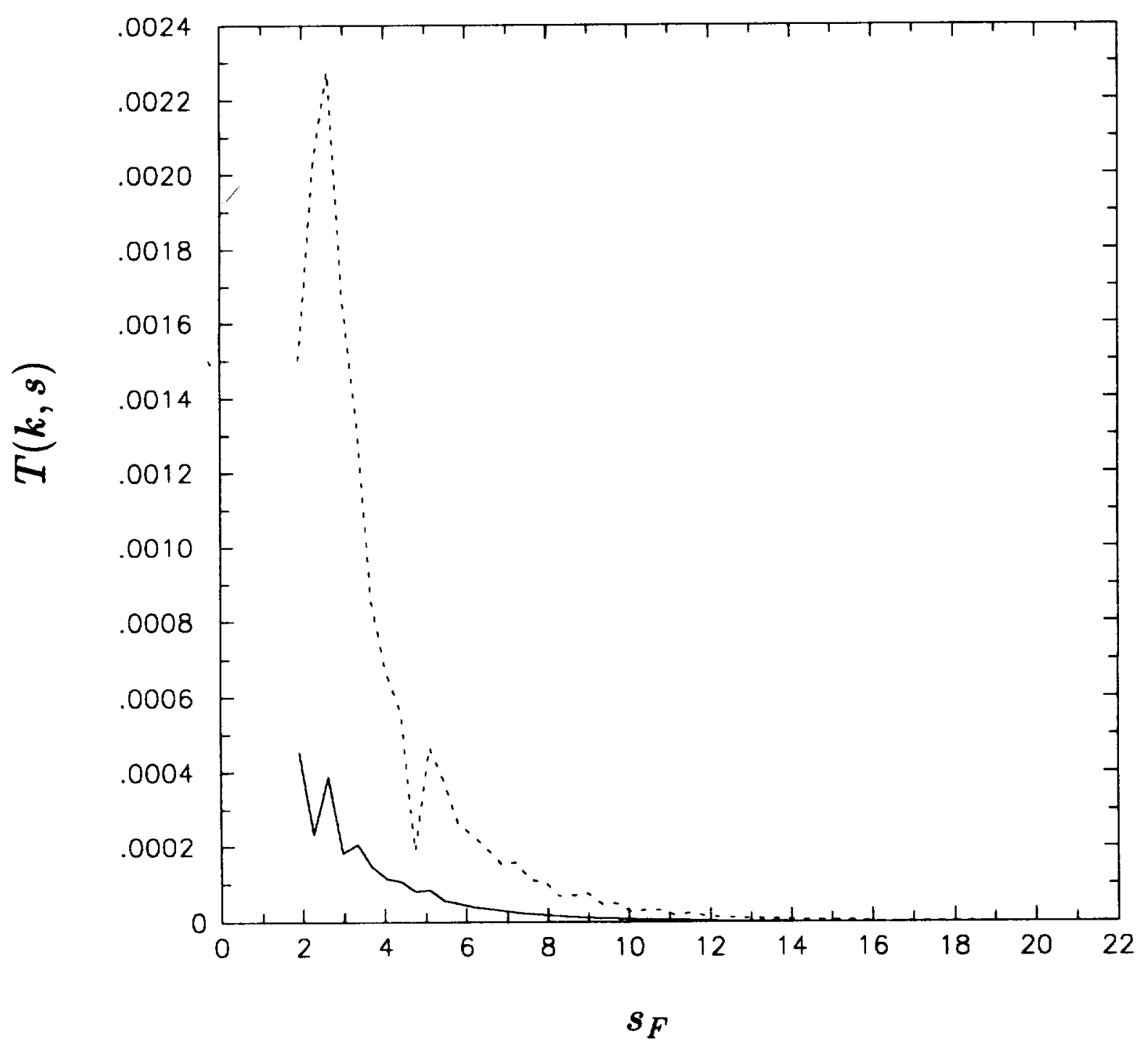


Fig. 13

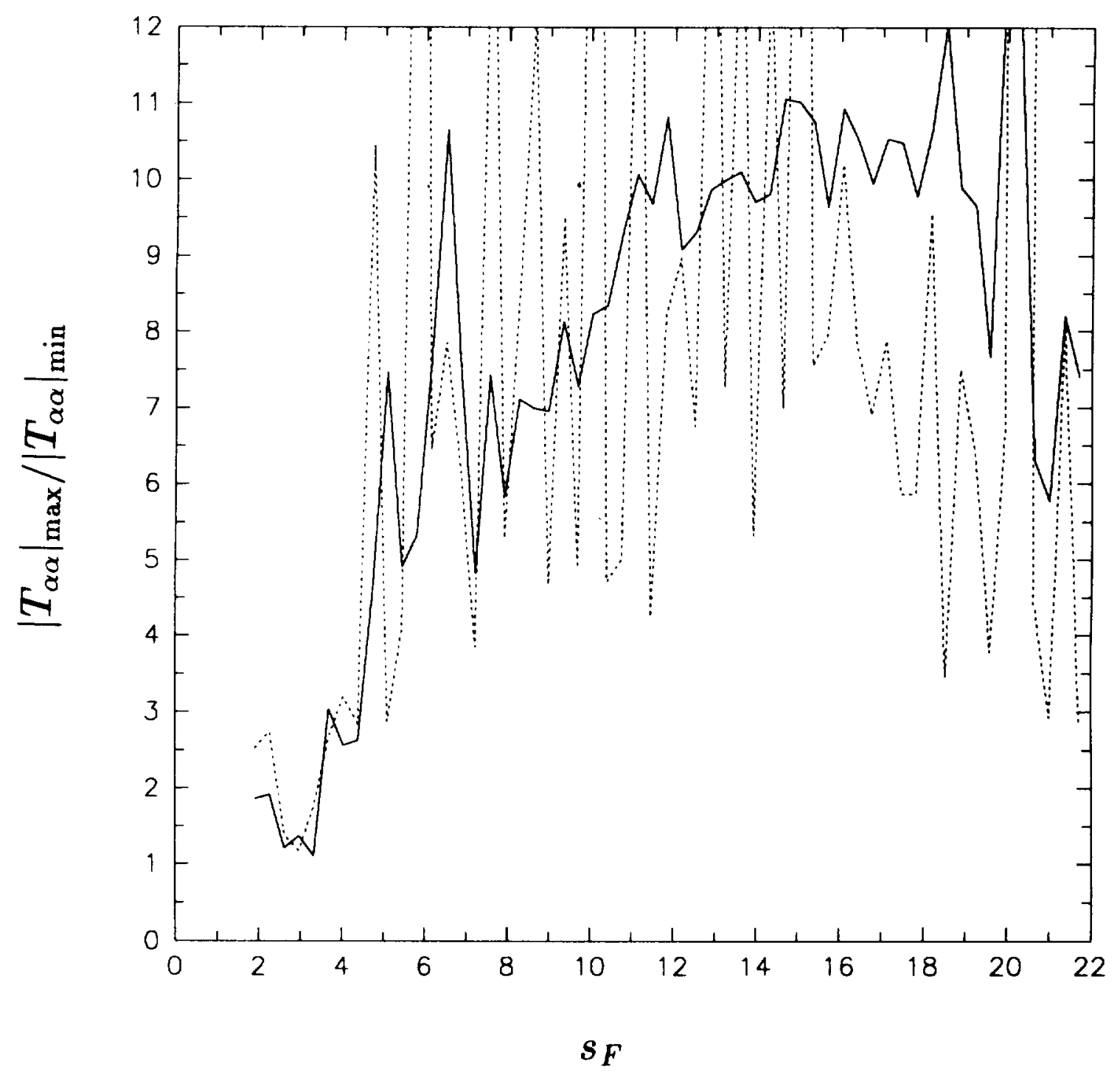


Fig. 14

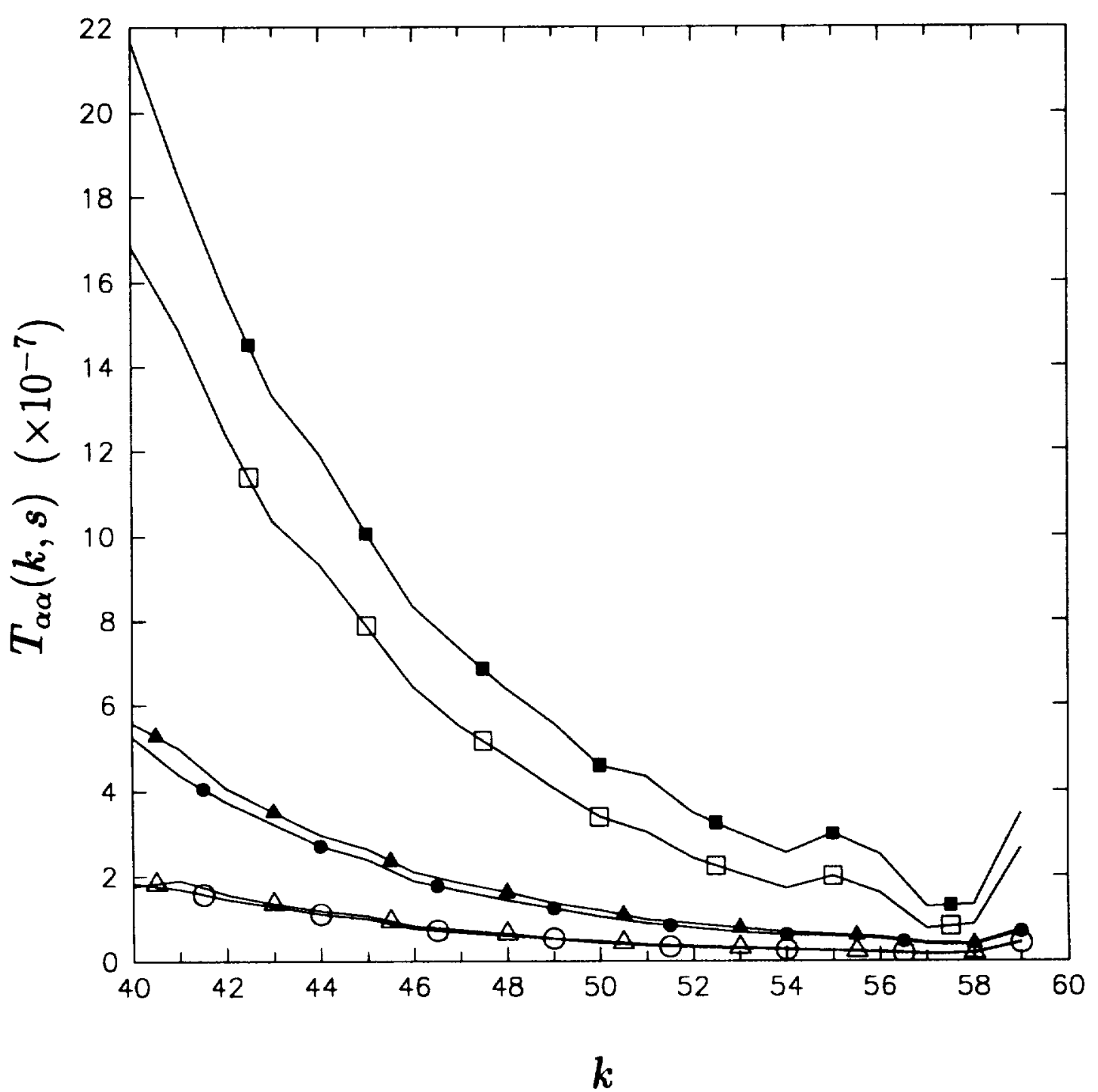


Fig. 15

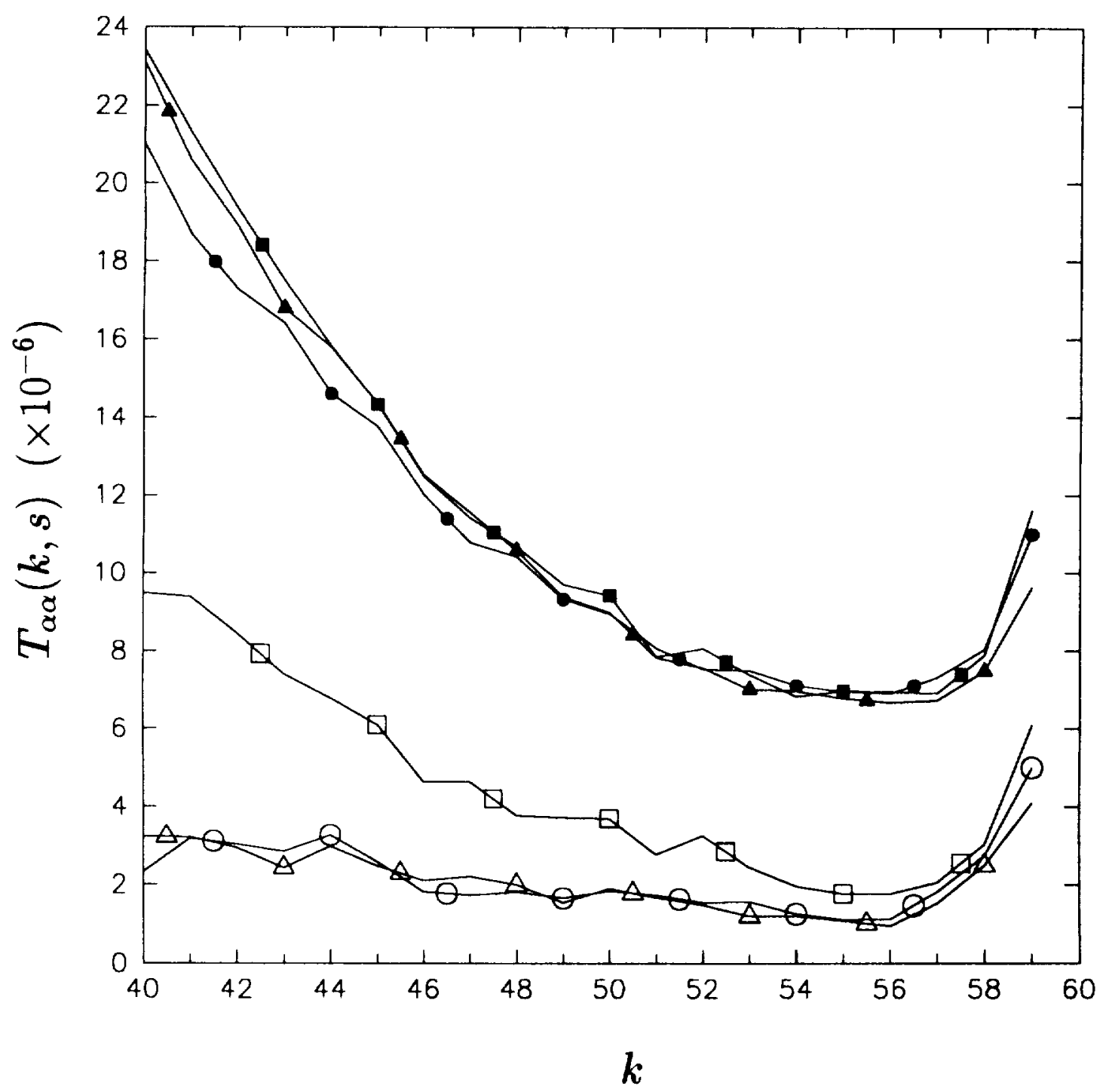




\begin{tabular}{|c|c|c|c|}
\hline \multicolumn{3}{|c|}{ REPORT DOCUMENTATION PAGE } & $\begin{array}{l}\text { Form Approved } \\
\text { OMB No. 0704-0188 }\end{array}$ \\
\hline \multicolumn{4}{|c|}{ 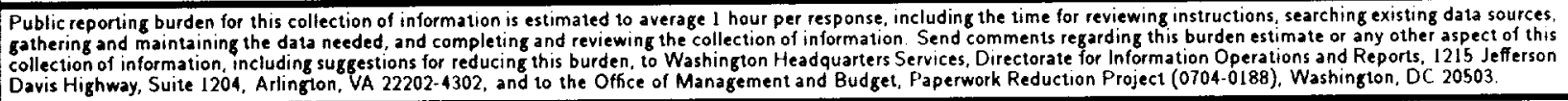 } \\
\hline 1. AGENCY USE ONLY(Leave blank) & $\begin{array}{l}\text { 2. REPORT DATE } \\
\text { August } 1994\end{array}$ & \multicolumn{2}{|c|}{$\begin{array}{l}\text { 3. REPORT TYPE ANO DATES COVERED } \\
\text { Contractor Report }\end{array}$} \\
\hline \multicolumn{3}{|c|}{$\begin{array}{l}\text { 4. TITLE AND SUBTITLE } \\
\text { SCALE DISPARITY ANDSPECTRAL TRANSFER IN ANISOTROPIC: } \\
\text { NUMERICAL TURBULENC: }\end{array}$} & \multirow[t]{2}{*}{$\begin{array}{l}\text { 5. FUNDING NUMBERS } \\
\text { C: NAS1-19480 } \\
\text { WU } 505-90-52-01\end{array}$} \\
\hline \multicolumn{3}{|l|}{$\begin{array}{l}\text { 6. AUTHOR(S) } \\
\text { Ye Zhou } \\
\text { P.K. Yeung } \\
\text { James G. Brasseur }\end{array}$} & \\
\hline \multicolumn{3}{|c|}{$\begin{array}{l}\text { 7. PERFORMING ORGANIZATION NAME(S) AND ADDRESS(ES) } \\
\text { Institute for Computer Applications in Science } \\
\text { and Engineering } \\
\text { Mail Stop } 132 \text { C, NASA Langley Research Center } \\
\text { Hampton, VA 23681-0001 }\end{array}$} & $\begin{array}{l}\text { 8. PERFORMING ORGANIZATION } \\
\text { REPORT NUMBER } \\
\text { ICASE Report No. } 94-64\end{array}$ \\
\hline \multicolumn{3}{|c|}{$\begin{array}{l}\text { 9. SPONSORING/MONITORING AGENCY NAME(S) AND ADDRESS(ES) } \\
\text { National Aeronautics and Space Administration } \\
\text { Langley Research Center } \\
\text { Hampton, VA } 23681-0001\end{array}$} & $\begin{array}{l}\text { 10. SPONSORING/MONITORING } \\
\text { AGENCY REPORT NUMBER } \\
\text { NASA CR-194956 } \\
\text { ICASE Report No. } 94-64\end{array}$ \\
\hline \multicolumn{4}{|c|}{$\begin{array}{l}\text { 11. SUPPLEMENTARY NOTES } \\
\text { Langley Technical Monitor: Michael F. Card } \\
\text { Final Report } \\
\text { Submitted to Physics of Fluids }\end{array}$} \\
\hline \multicolumn{2}{|c|}{$\begin{array}{l}\text { 12a. DISTRIBUTION/AVAILABILITY STATEMENT } \\
\text { Unclassified-Unlimited } \\
\text { Subject Category } 34\end{array}$} & & 12b. DISTRIBUTION CODE \\
\hline \multicolumn{4}{|c|}{$\begin{array}{l}\text { 13. ABSTRACT (Maximum } 200 \text { words) } \\
\text { To study the effect of cancelations within long-range interactions on local isotropy at the small scales (Waleffe, Phys. } \\
\text { Fluids } A, \mathbf{4}, 1992 \text { ), we calculate explicitly the degree of cancelation in distant triadic interactions in the simulations } \\
\text { of Yeung \& Brasseur (Phys. Fluids } A, 3,1991) \text { and Yeung, Brasseur \& Wang (to appear, J. Fluid Mech.) using } \\
\text { the single scale disparity parameter " } s \text { " developed by Zhou (Phys. Fluids } A, 5,1993 \text { ). In the simulations, initially } \\
\text { isotropic simulated turbulence was subjected to coherent anisotropic forcing at the large scales and the smallest } \\
\text { scales were found to become anisotropic as a consequence of direct large-small scale couplings. We find that the } \\
\text { marginally distant interactions in the simulation do not cancel out under summation and that the development of } \\
\text { small-scale anisotropy is indeed a direct consequence of the distant triadic group, as argued by Yeung, et. al . A } \\
\text { reduction of anisotropy at later times occurs as a result of the isotropizing influences of more local energy-cascading } \\
\text { triadic interactions. Nevertheless, the local-to-nonlocal triadic group persists as an anisotropizing influence at later } \\
\text { times. We find that, whereas long-range interactions, in general, contribute little to net energy transfer into or out } \\
\text { of a high wavenumber shell } k \text {, the anisotropic transfer of component energy within the shell increases with increasing } \\
\text { scale separation } s \text {. These results are consistent with results by Zhou, and Brasseur \& Wei (Phys. Fluids, } 6 \text {, } 1994 \text { ), } \\
\text { and suggest that the anisotropizing influences of long range interactions should persist to higher Reynolds numbers. } \\
\text { The residual effect of the forced distant triadic group in this low-Reynolds number simulation is found to be forward } \\
\text { cascading, on average. }\end{array}$} \\
\hline \multirow{2}{*}{\multicolumn{2}{|c|}{$\begin{array}{l}\text { 14. SUBJECT TERMS } \\
\text { anisotropic turbulence, scaling interaction, spectral energy }\end{array}$}} & transfer & $\begin{array}{l}\text { 15. NUMBER OF PAGES } \\
35\end{array}$ \\
\hline & & & $\begin{array}{c}\text { 16. PRICE CODE } \\
\text { A03 }\end{array}$ \\
\hline $\begin{array}{l}\text { 17. SECURITY CLASSIFICATION } \\
\text { OF REPORT } \\
\text { Unclassified }\end{array}$ & $\begin{array}{l}\text { 18. SECURITY CLASSIFICATION } \\
\text { OF THIS PAGE } \\
\text { Unclassified }\end{array}$ & $\begin{array}{l}\text { 19. SECURITY CLASSIFICATION } \\
\text { OF ABSTRACT }\end{array}$ & $\begin{array}{l}\text { 20. LIMITATION } \\
\text { OF ABSTRACT }\end{array}$ \\
\hline NSN 7540-01-280-5500 & & & $\begin{array}{l}\text { Standard Form 298(Rev. 2-89) } \\
\text { Prescribed by ANSI Sid. 239-18 } \\
\text { 298-102 }\end{array}$ \\
\hline
\end{tabular}

